\title{
TYPE Ia SUPERNOVAE STRONGLY INTERACTING WITH THEIR CIRCUMSTELLAR MEDIUM
}

\author{
Jeffrey M. Silverman ${ }^{1,2}$, Peter E. Nugent ${ }^{3}$, Avishay Gal-Yam ${ }^{4}$, Mark Sullivan ${ }^{5}$, D. Andrew Howell ${ }^{6,7}$, \\ Alexei V. Filippenko ${ }^{2}$, Iair Arcavi ${ }^{4}$, Sagi Ben-Ami ${ }^{4}$, Joshua S. Bloom ${ }^{2}$, S. Bradley Cenko $^{2}$, Yi CaO ${ }^{8}$, Ryan Chornock $^{9}$, \\ Kelsey I. Clubb ${ }^{2}$, Alison L. Coil ${ }^{10}$, Ryan J. Foley ${ }^{9,21}$, Melissa L. Graham ${ }^{6,7}$, Christopher V. Griffith ${ }^{11}$, Assaf Horesh ${ }^{8}$, \\ Mansi M. Kasliwal ${ }^{12}$, Shrinivas R. Kulkarni ${ }^{8}$, Douglas C. LeOnard ${ }^{13}$, Weidong Li ${ }^{2,22}$, Thomas Matheson ${ }^{14}$, \\ Adam A. Miller ${ }^{2}$, Maryam Modjaz ${ }^{15}$, Eran O. Ofek ${ }^{4}$, Yen-Chen Pan ${ }^{16}$, Daniel A. Perley ${ }^{9}$, Dovi Poznanski ${ }^{17}$, \\ Robert M. Quimby ${ }^{18}$, Thea N. Steele ${ }^{2,19}$, Assaf Sternberg ${ }^{20}$, Dong Xu $^{4}$, ANd Ofer Yaron ${ }^{4}$ \\ ${ }^{1}$ Department of Astronomy, University of Texas, Austin, TX 78712-0259, USA; jsilverman@astro.as.utexas.edu \\ 2 Department of Astronomy, University of California, Berkeley, CA 94720-3411, USA \\ ${ }^{3}$ Lawrence Berkeley National Laboratory, Berkeley, CA 94720, USA \\ ${ }^{4}$ Benoziyo Center for Astrophysics, The Weizmann Institute of Science, Rehovot 76100, Israel \\ ${ }^{5}$ School of Physics and Astronomy, University of Southampton, Southampton SO17 1BJ, UK \\ ${ }^{6}$ Las Cumbres Observatory Global Telescope Network, Goleta, CA 93117, USA \\ ${ }^{7}$ Department of Physics, University of California, Santa Barbara, CA 93106, USA \\ ${ }^{8}$ Cahill Center for Astrophysics, California Institute of Technology, Pasadena, CA 91125, USA \\ ${ }_{9}^{9}$ Harvard-Smithsonian Center for Astrophysics, Cambridge, MA 02138, USA \\ ${ }^{10}$ Department of Physics, University of California, San Diego, La Jolla, CA 92093, USA \\ ${ }^{11}$ Department of Astronomy and Astrophysics, The Pennsylvania State University, University Park, PA 16802, USA \\ 12 Observatories of the Carnegie Institution of Science, Pasadena, CA 91101, USA \\ ${ }^{13}$ Department of Astronomy, San Diego State University, San Diego, CA 92182-1221, USA \\ ${ }^{14}$ National Optical Astronomy Observatory, Tucson, AZ 85719-4933, USA \\ ${ }^{15}$ Center for Cosmology and Particle Physics, Department of Physics, New York University, New York, NY 10003, USA \\ ${ }^{16}$ Department of Physics (Astrophysics), University of Oxford, Keble Road, Oxford OX1 3RH, UK \\ ${ }^{17}$ School of Physics and Astronomy, Tel Aviv University, Tel Aviv, Israel \\ ${ }^{18}$ Kavli IPMU, The University of Tokyo, Kashiwanoha 5-1-5, Kashiwa 277-8583, Japan \\ ${ }^{19}$ Department of Computer Science, Kutztown University of Pennsylvania, Kutztown, PA 19530, USA \\ ${ }^{20}$ Max-Planck-Institut für Astrophysik, D-85741 Garching, Germany \\ Received 2013 March 29; accepted 2013 April 26; published 2013 June 14
}

\begin{abstract}
Owing to their utility for measurements of cosmic acceleration, Type Ia supernovae (SNe Ia) are perhaps the beststudied class of SNe, yet the progenitor systems of these explosions largely remain a mystery. A rare subclass of SNe Ia shows evidence of strong interaction with their circumstellar medium (CSM), and in particular, a hydrogenrich CSM; we refer to them as SNe Ia-CSM. In the first systematic search for such systems, we have identified 16 SNe Ia-CSM, and here we present new spectra of 13 of them. Six SNe Ia-CSM have been well studied previously, three were previously known but are analyzed in depth for the first time here, and seven are new discoveries from the Palomar Transient Factory. The spectra of all SNe Ia-CSM are dominated by H $\alpha$ emission (with widths of $\sim 2000 \mathrm{~km} \mathrm{~s}^{-1}$ ) and exhibit large $\mathrm{H} \alpha / \mathrm{H} \beta$ intensity ratios (perhaps due to collisional excitation of hydrogen via the SN ejecta overtaking slower-moving CSM shells); moreover, they have an almost complete lack of He I emission. They also show possible evidence of dust formation through a decrease in the red wing of H $\alpha$ 75-100 days past maximum brightness, and nearly all SNe Ia-CSM exhibit strong Na I D absorption from the host galaxy. The absolute magnitudes (uncorrected for host-galaxy extinction) of SNe Ia-CSM are found to be $-21.3 \mathrm{mag} \leqslant M_{R} \leqslant-19 \mathrm{mag}$, and they also seem to show ultraviolet emission at early times and strong infrared emission at late times (but no detected radio or X-ray emission). Finally, the host galaxies of SNe Ia-CSM are all late-type spirals similar to the Milky Way, or dwarf irregulars like the Large Magellanic Cloud, which implies that these objects come from a relatively young stellar population. This work represents the most detailed analysis of the SN Ia-CSM class to date.
\end{abstract}

Key words: circumstellar matter - supernovae: general - supernovae: individual (SN 1997cy, SN 1999E, SN 2002ic, SN 2005gj, SN 2008J, SN 2008cg, SN 2011jb, CSS120327:110520-015205, PTF11kx, PTF10htz, PTF10iuf, PTF10yni, PTF11dsb, PTF11hzx, PTF12efc)

\section{INTRODUCTION}

While Type Ia supernovae ( $\mathrm{SNe}$ Ia) have been used as precise distance indicators for nearly two decades (Phillips 1993), the nature of their progenitor systems and explosion mechanisms is still unclear (see Howell 2011 for further information). While there is general acceptance that they are the result of the thermonuclear explosion of $\mathrm{C} / \mathrm{O}$ white dwarfs (WDs), it is now

\footnotetext{
${ }^{21}$ Clay Fellow

22 Deceased 2011 December 12.
}

likely that there are at least two major channels that lead to an SN Ia. The single-degenerate (SD) channel occurs when the WD accretes matter from a nondegenerate companion star (e.g., Whelan \& Iben 1973), while the double-degenerate (DD) channel is the result of the merger of two WDs (e.g., Iben \& Tutukov 1984; Webbink 1984).

Extremely nearby $\mathrm{SNe}$ Ia which were discovered soon after explosion have recently led to tight constraints on the size and luminosity of the companion star, thus ruling out many plausible SD scenarios for these objects (e.g., Nugent et al. 2011; Ganeshalingam et al. 2011; Brown et al. 2012; Foley et al. 
2012a; Bloom et al. 2012; Silverman et al. 2012b). In addition, the so-called super-Chandrasekhar mass SNe Ia are thought to contain $>1.4 M_{\odot}$ of SN ejecta and thus are likely formed from the DD scenario (e.g., Howell et al. 2006; Yamanaka et al. 2009; Scalzo et al. 2010; Silverman et al. 2011; Taubenberger et al. 2011). However, there are a few SNe Ia that show strong evidence for the SD channel, possibly with a red giant (RG) companion. Photoionization and subsequent recombination of circumstellar medium (CSM) has been observed in relatively normal SNe Ia (Patat et al. 2007; Blondin et al. 2009; Simon et al. 2009), and CSM has been detected in the spectra of at least $20 \%$ of SNe Ia in spiral galaxies (Sternberg et al. 2011) and has been linked to SN Ia explosion properties (Foley et al. 2012b).

Even greater interaction with CSM has been seen in a small number of SNe Ia whose spectra contain strong, narrow hydrogen emission and whose luminosities often exceed those of the more "typical" SNe Ia that follow the light-curve decline rate versus peak luminosity correlation (i.e., the "Phillips relation"; Phillips 1993). These "hybrid" objects resemble Type IIn $\mathrm{SNe}$ (SNe IIn) and have been dubbed SNe Ia/IIn, Ian, IIa, and IIan. Under the standard SN classification scheme (e.g., Filippenko 1997), any SN with hydrogen features in its optical spectrum is considered an SN II and the subset of these showing relatively narrow emission lines are referred to as $\mathrm{SNe}$ IIn. Objects with relatively narrow hydrogen emission lines that are further linked (spectroscopically) to SNe Ia could be denoted "Type IIna," though this moniker is somewhat cumbersome and obfuscating. Therefore, in this work, we choose to label such events "SNe Ia-CSM" (or sometimes as "Ia-CSM objects") to highlight the connection to the physically distinct Type Ia class.

SNe Ia-CSM have spectra which appear to be "diluted" SN Ia spectra with the aforementioned narrow hydrogen emission lines superposed (leading to the SN IIn resemblance). Their light curves are broad and quite long-lived, peaking at absolute magnitudes brighter than about -19 mag. The two best-studied objects in this class are SNe 2002ic and 2005gj (Hamuy et al. 2003; Deng et al. 2004; Kotak et al. 2004; Wang et al. 2004; Wood-Vasey et al. 2004; Aldering et al. 2006; Prieto et al. 2007). Until recently there was some debate in the literature as to whether these objects are truly $\mathrm{SNe}$ Ia or are in fact some strange flavor of core-collapse SNe (CCSNe; e.g., Benetti et al. 2006; Trundle et al. 2008).

This possible controversy seems to have been settled by the discovery and analysis of PTF11kx (Dilday et al. 2012; Silverman et al. 2013). This object was discovered by the Palomar Transient Factory (PTF; Rau et al. 2009; Law et al. 2009) and shown to initially resemble the somewhat overluminous Type Ia SN 1999aa (Li et al. 2001; Strolger et al. 2002; Garavini et al. 2004). Optical spectra of PTF11 kx soon developed a strong $\mathrm{H} \alpha$ feature with a P-Cygni profile, eventually resembling spectra of SNe 2002ic and 2005gj, the previously mentioned best-studied SNe Ia-CSM. Using early-time data, Dilday et al. (2012) find that PTF11kx was a bona fide SN Ia with a symbiotic nova progenitor (i.e., an SD scenario). Analyzing late-time data, Silverman et al. (2013) present further evidence that PTF11kx was an SN Ia that is strongly interacting with multiple thin CSM shells. These findings can logically be extended to imply that all $\mathrm{SNe}$ Ia-CSM are likely to be real SNe Ia with significant amounts of H-rich CSM, possibly caused in part by a nondegenerate companion.

In Section 2, we discuss our search for and identification (or reidentification) of previously known SNe Ia-CSM. Most of these have not been studied in much detail before, and we present new spectra of many of them. Similarly, in Section 3, we discuss new SNe Ia-CSM discovered by PTF. Section 4 contains the analysis of our spectra of all SNe Ia-CSM presented herein, in addition to the spectra of PTF11kx from Dilday et al. (2012) and Silverman et al. (2013). Finally, we recap our conclusions in Section 5 and summarize the major observational characteristics shared by all SNe Ia-CSM.

\section{PREVIOUSLY KNOWN SNe Ia-CSM}

In order to find SNe Ia-CSM that may have previously been classified as SNe IIn, we use the SuperNova IDentification code (SNID; Blondin \& Tonry 2007). SNID classifies SN spectra by cross-correlating an input spectrum with a large database of observed SN spectra (known as "templates"). To identify SNe Ia-CSM, we created a special set of spectral templates that consisted of all 310 spectra of 163 objects classified as SNe IIn from the Berkeley SN Group's database (Silverman et al. 2012a). In addition to these, we also included templates of a handful of underluminous, overluminous, and normal SNe Ia at a variety of epochs, as well as 27 spectral templates of the well-known Ia-CSM objects SNe 2002ic and 2005gj (mentioned in Section 1).

We then ran all of our spectra of PTF $11 \mathrm{kx}$ and the previously known Ia-CSM objects SNe 1999E, 2002ic, and 2005gj (see Sections 2.1 and 2.2) through SNID using this newly created template set, making sure to ignore any templates made from the object currently under consideration. The best-matching templates for each of these known SNe Ia-CSM were visually inspected, and SNe which were often found in the top 10-20 best-matching templates were flagged for further examination. This yielded 18 possible SNe Ia-CSM. Upon deeper analysis, it was found that many of the spectra matched those of SNe IaCSM and "normal" SNe IIn equally well; also, spectra of some objects had very low signal-to-noise ratios. While some of these may perhaps be true SNe Ia-CSM, we cannot confidently claim this for most of them. Thus, after this closer inspection was conducted, 4 of the 18 possible SNe Ia-CSM are convincingly part of the SN Ia-CSM class.

Interestingly, all four of our "newly discovered" members of the SN Ia-CSM class were noted to be similar to the prototypical Ia-CSM objects SNe 2002ic and 2005gj, but only in unrefereed spectral classifications. From a literature search, no mention of these objects as SNe Ia-CSM is found anywhere else, except for SN 2008J which was studied by Taddia et al. (2012), work that was made public during the final stages of preparing this manuscript. Adding these four objects to the prototypical SNe 2002ic and 2005gj and to the less-studied but still fairly well-known Ia-CSM objects SNe 1997cy and 1999E gives us a sample size of eight SNe Ia-CSM out of the hundreds of $\mathrm{SNe}$ IIn discovered in the last $\sim 15 \mathrm{yr}$. Tables 1 and 2 give basic information for all eight of these SNe Ia-CSM and their host galaxies, respectively, and our spectra of six of these $\mathrm{SNe}$ are summarized in Table 3. Upon publication, all spectra presented in this paper will be available in electronic format on the Weizmann Interactive Supernova data REPository (WISeREP Yaron \& Gal-Yam 2012). ${ }^{23}$ These eight SNe Ia-CSM are briefly discussed (in pairs) below.

\footnotetext{
23 http://www.weizmann.ac.il/astrophysics/wiserep.
} 
Table 1

Non-PTF SNe Ia-CSM

\begin{tabular}{|c|c|c|c|c|c|c|}
\hline SN Name & $\begin{array}{c}\text { Discovery } \\
\text { Date }\end{array}$ & $\begin{array}{l}\text { Approx. Date of } \\
\text { Maximum }\end{array}$ & $\begin{array}{l}\text { Discovery } \\
\text { Reference }\end{array}$ & $\begin{array}{c}\text { Classification } \\
\text { Reference }^{\mathrm{a}}\end{array}$ & $\begin{array}{c}\text { Peak Absolute } \\
\text { Magnitude }^{\mathrm{b}}\end{array}$ & $\begin{array}{c}\text { Galactic } \\
\text { Reddening }\end{array}$ \\
\hline SN 1997cy & 1997 Jul 16 & $\ldots{ }^{d}$ & IAUC 6706 & T00, G00 & $<-20.1(V)$ & 0.021 \\
\hline SN 1999E & 1999 Jan 15 & $\ldots{ }^{d}$ & IAUC 7089 & IAUC 7090, R03 & $<-19.5(V)$ & 0.088 \\
\hline SN 2002ic & 2002 Nov 13 & 2002 Nov 27 & IAUC 8019 & IAUC 8028 & $<-20.3(V)$ & 0.059 \\
\hline SN 2005gj & 2005 Sep 26 & 2005 Oct 14 & CBET 247 & CBET 302 & $-20.2(g)$ & 0.121 \\
\hline SN 2008J & 2008 Jan 15 & 2008 Feb 3 & CBET 1211 & CBET 1218 & $-19.2(r)$ & 0.023 \\
\hline SN 2008cg & 2008 May 5 & 2008 Apr 29 & CBET 1366 & CBET 1420 & -19.4 (unf) & 0.050 \\
\hline SN $2011 \mathrm{jb}$ & 2011 Nov 28 & $\ldots d$ & CBET 2947 & CBET 2947 & $-20.3(R)$ & 0.034 \\
\hline CSS120327:110520-015205 & $2012 \operatorname{Mar} 27$ & $2012 \operatorname{Mar} 8$ & ATel 4081 & ATel 4081 & -20.5 (unf) & 0.052 \\
\hline
\end{tabular}

Notes.

a T00, Turatto et al. (2000); G00, Germany et al. (2000); R03, Rigon et al. (2003).

b The optical band of the peak absolute magnitude (and approximate date of maximum brightness) is given in parentheses; unf: unfiltered.

${ }^{c}$ Galactic reddening toward each SN as derived from the dust maps of Schlegel et al. (1998), including the corrections of Peek \& Graves (2010).

${ }^{\mathrm{d}} \mathrm{SN}$ was discovered after maximum brightness.

Table 2

Non-PTF Ia-CSM Host Galaxies

\begin{tabular}{lll}
\hline \hline SN Name & \multicolumn{1}{c}{ Name } & Type \\
\hline SN 1997cy & Sersic 040/06:[GGP90] 342 & Dwarf irregular \\
SN 1999E & GSC 6116 00964 & Late-type spiral \\
SN 2002ic & NEAT J013002.81+215306.9 & 0.0258 \\
SN 2005gj & SDSS J030111.99-003313.9 & Late-type spiral (Sbc) \\
SN 2008J & MCG -02-07-033 & Dwarf irregular \\
SN 2008cg & FGC 1965 & Late-type spiral (SBbc) \\
SN 2011jb & SDSS J113704.81+152813.9 & Late-type spiral (Scd) \\
CSS120327:110520-015205 & SDSS J110520.10-015204.9 & Dwarf irregular \\
\hline
\end{tabular}

\subsection{SN 2002ic and SN $2005 \mathrm{gj}$}

As already mentioned, the two prototypical and best-studied SNe Ia-CSM are SN 2002ic (Hamuy et al. 2003; Deng et al. 2004; Kotak et al. 2004; Wang et al. 2004; Wood-Vasey et al. 2004) and SN 2005gj (Aldering et al. 2006; Prieto et al. 2007). The photometric and spectral evolution of these two objects has been well studied in the works cited above. Both were found to be more luminous than typical SNe Ia and most SNe IIn, have spectra consisting of relatively narrow $\mathrm{H} \alpha$ emission (with P-Cygni profiles) on top of a "diluted" SN Ia spectrum, and broad, slowly evolving light curves. To the already impressive data on SN 2005gj in the literature, we add five spectra (three at early times and two at very late times) that were originally published by Silverman et al. (2012a) and are displayed in Figure 1.

\subsection{SN 1997cy and SN 1999E}

The two next-best-studied SNe Ia-CSM are SN 1997cy (Turatto et al. 2000; Germany et al. 2000) and SN 1999E (Filippenko 2000; Rigon et al. 2003). Neither of these objects have as much data as either SNe 2002 ic or $2005 \mathrm{gj}$, but they have still been studied fairly rigorously due to their peculiar nature (especially before the discovery of SNe 2002ic and 2005gj). Again, both objects showed relatively bright absolute magnitudes with spectra that somewhat resembled those of $\mathrm{SNe}$ Ia with superposed $\mathrm{H} \alpha$ emission. In Figure 2, we plot our six spectra of SN 1999E, which were first published by Filippenko (2000), before it was realized that this was an SN Ia. Unfortunately, it seems that SN 1999E was discovered well after maximum brightness; thus, the phases displayed in Figure 2 are relative to the UT date of discovery (1999 January 15; Rigon et al. 2003), and are likely from a much later phase if calculated relative to maximum brightness.

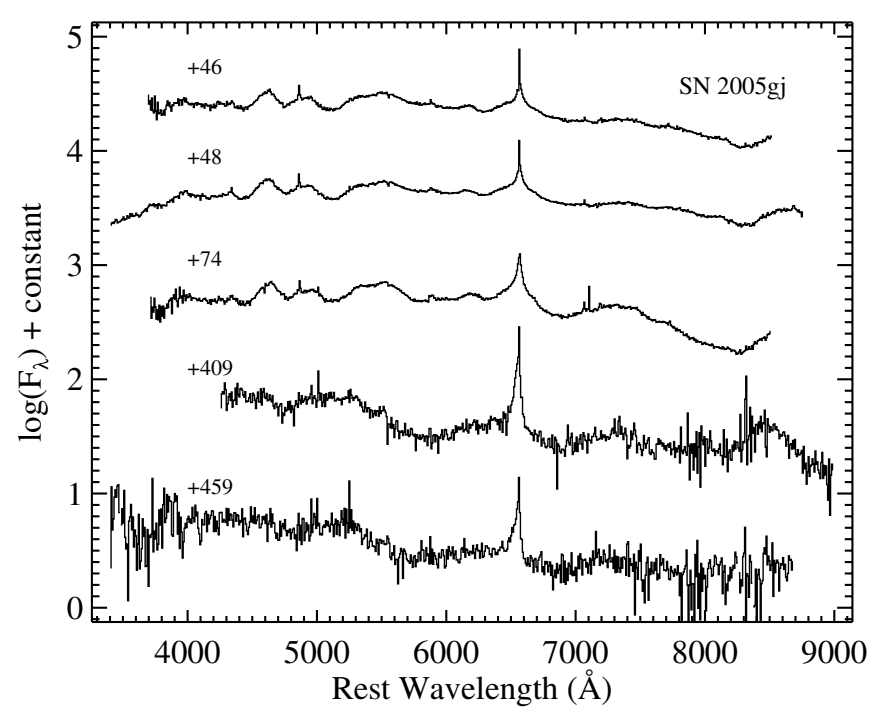

Figure 1. Spectra of SN 2005gj, originally published by Silverman et al. (2012a), labeled with age relative to maximum brightness. The data have had their host-galaxy recession velocity removed and have been corrected for Galactic reddening.

\subsection{SN 2008J and SN $2008 \mathrm{cg}$}

$\mathrm{SNe} 2008 \mathrm{~J}$ and $2008 \mathrm{cg}$ were both claimed to resemble SNe 2002ic and 2005gj in unrefereed classification circulars (Stritzinger et al. 2008; Filippenko et al. 2008), and very recently Taddia et al. (2012) have published an analysis of SN 2008J. Fox et al. (2011) investigated the $\mathrm{H} \alpha$ profiles and presented midinfrared (IR) photometry from a Spitzer/IRAC survey of these objects (referred to as simply "SNe IIn") and found that both 
Table 3

Spectra of Non-PTF SNe Ia-CSM

\begin{tabular}{|c|c|c|c|c|c|}
\hline UT Date & $\begin{array}{l}\text { Age }^{a} \\
\text { (days) }\end{array}$ & Instrument $^{\mathrm{b}}$ & $\begin{array}{c}\text { Range } \\
(\AA)\end{array}$ & $\begin{array}{c}\text { Res. }^{c} \\
(\AA)\end{array}$ & $\begin{array}{c}\text { Exp. } \\
\text { (s) }\end{array}$ \\
\hline \multicolumn{6}{|c|}{ SN $1999 E^{d}$} \\
\hline 1999 Jan 19.7 & 4 & LRIS & $5120-8850$ & 7 & 200 \\
\hline 1999 Jan 20.7 & 5 & LRIS & $3760-6240$ & 4.5 & 200 \\
\hline 1999 Jan 21.7 & 6 & LRIS & $6450-10200$ & 7 & 300 \\
\hline 1999 Feb 12.5 & 27 & Kast & $3382-10500$ & $6 / 11$ & 1800 \\
\hline 1999 Feb 23.5 & 38 & Kast & $3366-10550$ & $6 / 11$ & 1200 \\
\hline 1999 Mar 12.5 & 55 & Kast & 3442-10466 & $6 / 11$ & 1800 \\
\hline \multicolumn{6}{|c|}{$\mathrm{SN} 2005 \mathrm{gj}^{\mathrm{e}}$} \\
\hline 2005 Dec 2.4 & 46 & DEIMOS & $3897-9070$ & 3 & 300 \\
\hline 2005 Dec 4.4 & 48 & LRIS & $3280-9320$ & $4.5 / 7$ & 300 \\
\hline 2006 Jan 1.4 & 74 & DEIMOS & 3918-9061 & 3 & 600 \\
\hline 2006 Dec 23.4 & 409 & DEIMOS & 4496-9574 & 3 & 1500 \\
\hline 2007 Feb 14.3 & 459 & LRIS & 3206-9238 & $6.4 / 7$ & 1200 \\
\hline \multicolumn{6}{|c|}{ SN 2008J } \\
\hline 2008 Feb 16.2 & 12 & Kast & $4440-10500$ & $4.9 / 11.9$ & 900 \\
\hline 2008 Aug 1.5 & 177 & Kast & $4292-10800$ & $6.0 / 11.0$ & 900 \\
\hline 2008 Aug 26.5 & 201 & Kast & $4232-10800$ & $6.7 / 12.3$ & 900 \\
\hline 2008 Sep 7.5 & 213 & Kast & $4050-10740$ & $7.4 / 12.3$ & 1200 \\
\hline 2008 Sep 22.5 & 228 & Kast & $3488-10344$ & $4.6 / 11.0$ & 1200 \\
\hline 2008 Oct 7.5 & 243 & Kast & $3554-10780$ & $4.4 / 11.0$ & 1200 \\
\hline 2008 Oct 22.4 & 258 & Kast & $3476-6400$ & 9.0 & 2400 \\
\hline 2008 Nov 20.3 & 286 & Kast & $3480-10000$ & $5.0 / 11.0$ & 1800 \\
\hline 2008 Nov 23.4 & 289 & Kast & $3420-8000$ & $4.8 / 5.0$ & 1200 \\
\hline \multicolumn{6}{|c|}{ SN 2008cg } \\
\hline 2008 May 8.4 & 9 & Kast & $3300-10800$ & $5.4 / 11.6$ & 1800 \\
\hline 2008 May 15.4 & 15 & Kast & $3300-10800$ & $5.5 / 11.7$ & 1800 \\
\hline 2008 Jun 29.3 & 59 & Kast & $3300-10790$ & $5.9 / 10.8$ & 1500 \\
\hline 2008 Jul 7.3 & 67 & Kast & $3306-10800$ & $6.0 / 11.3$ & 1500 \\
\hline 2008 Aug 27.3 & 116 & LRIS & $3268-9240$ & $4.5 / 7.0$ & 454 \\
\hline \multicolumn{6}{|c|}{ SN 2011jb } \\
\hline 2011 Dec 24.4 & 24 & Kast & $3518-10138$ & $4.7 / 9.8$ & 2400 \\
\hline 2012 Jun 16.4 & 186 & LRIS & $3350-10152$ & $3.6 / 6.1$ & 600 \\
\hline 2012 Jul 16.3 & 213 & LRIS & 3727-9939 & $3.7 / 6.4$ & 600 \\
\hline \multicolumn{6}{|c|}{ CSS120327:110520-015205 } \\
\hline 2012 May 17.2 & 64 & LRIS & $3343-10100$ & $3.7 / 6.2$ & 300 \\
\hline 2012 Jun 16.3 & 92 & LRIS & 3346-10069 & $4.1 / 6.1$ & 1200 \\
\hline
\end{tabular}

\section{Notes.}

a Rest-frame days relative to maximum brightness, except for SNe 1999E and $2011 \mathrm{jb}$ where the epoch is relative to the UT date of discovery (1999 January 15 and 2011 November 28, respectively). See Table 1 for the dates of maximum brightness for the rest of the objects.

b LRIS: Low Resolution Imaging Spectrometer on the Keck 10 m telescope; Kast: Kast double spectrograph on the Shane $3 \mathrm{~m}$ telescope at Lick Observatory; DEIMOS: DEep Imaging Multi-Object Spectrograph on the Keck $10 \mathrm{~m}$ telescope.

c Approximate FWHM intensity resolution. If two numbers are listed, they represent the blue-side and red-side resolutions, respectively.

d These spectra of SN 1999E have been previously published by Filippenko (2000).

e These spectra of SN 2005gj have been previously published by Silverman et al. (2012a).

SNe were clearly detected in the mid-IR $\sim 500-600$ days after discovery (see Section 4.3 for more information). SN 2008J resembles SN 2005gj in an optical spectrum obtained only 2 days after discovery (Stritzinger et al. 2008), which is $\sim 2.5$ weeks before $r$-band maximum brightness (Taddia et al. 2012), and it continues to resemble SNe Ia-CSM in all nine of our

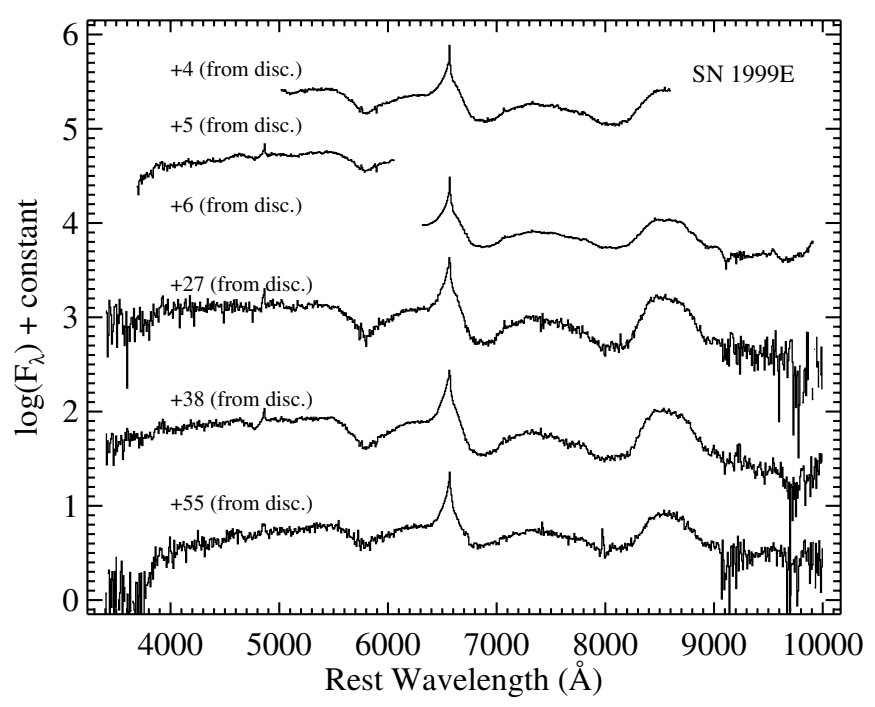

Figure 2. Spectra of SN 1999E, originally published in Filippenko (2000), labeled with age relative to the discovery date. The data have had their host-galaxy recession velocity removed and have been corrected for Galactic reddening.

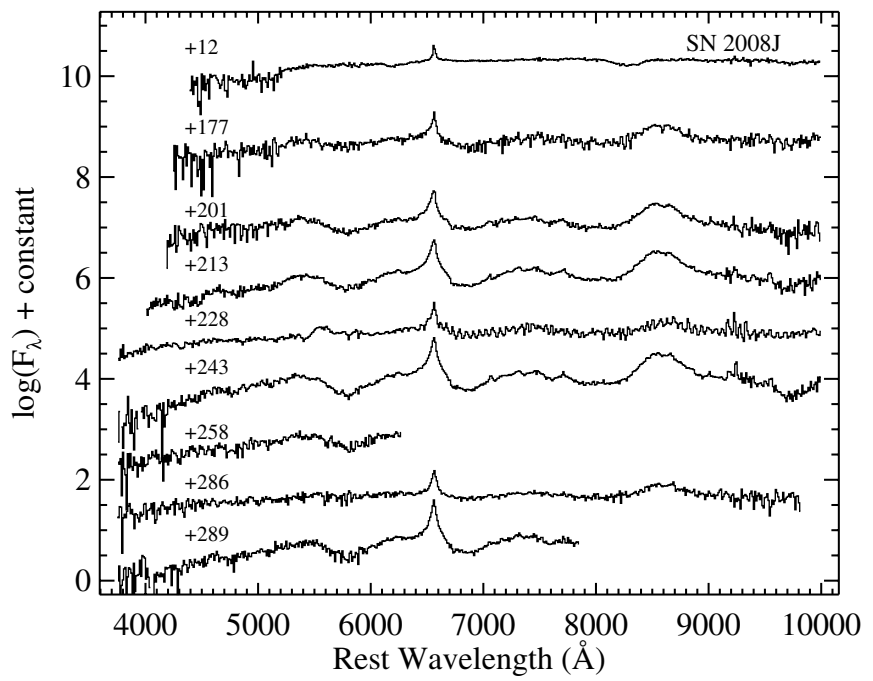

Figure 3. Spectra of SN 2008J, the H $\alpha$ profiles of which were analyzed by Fox et al. (2011), labeled with age relative to maximum brightness. The data have had their host-galaxy recession velocity removed and have been corrected for Galactic reddening.

spectra (plotted in Figure 3). Taddia et al. (2012) also found that the optical and near-IR spectra of SN 2008J show narrow H Balmer, Paschen, and Brackett emission (most with P-Cygni profiles) and that the optical spectra could be decomposed into a low-order polynomial continuum and the spectrum of the overluminous Type Ia SN 1991T. On the other hand, an optical spectrum of SN $2008 \mathrm{cg}$ resembles a relatively normal SN IIn 3 days after discovery (Blondin \& Calkins 2008), which is about 10 days past maximum brightness. However, by $\sim 2$ months past discovery, SN 2008cg was found to closely resemble SNe 2002ic and 2005gj (Filippenko et al. 2008). Our five spectra of this objects are shown in Figure 4. Note that the data on SNe 2008J and $2008 \mathrm{cg}$ presented here are the same optical spectra discussed by Fox et al. (2011). 


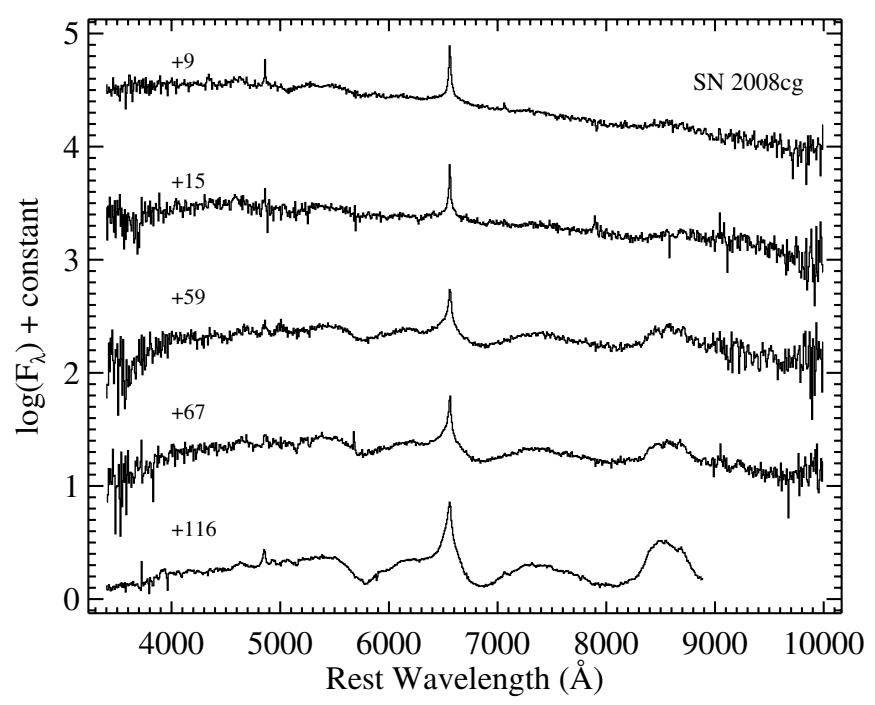

Figure 4. Spectra of SN $2008 \mathrm{cg}$, the $\mathrm{H} \alpha$ profiles of which were analyzed by Fox et al. (2011), labeled with age relative to maximum brightness. The data have had their host-galaxy recession velocity removed and have been corrected for Galactic reddening.

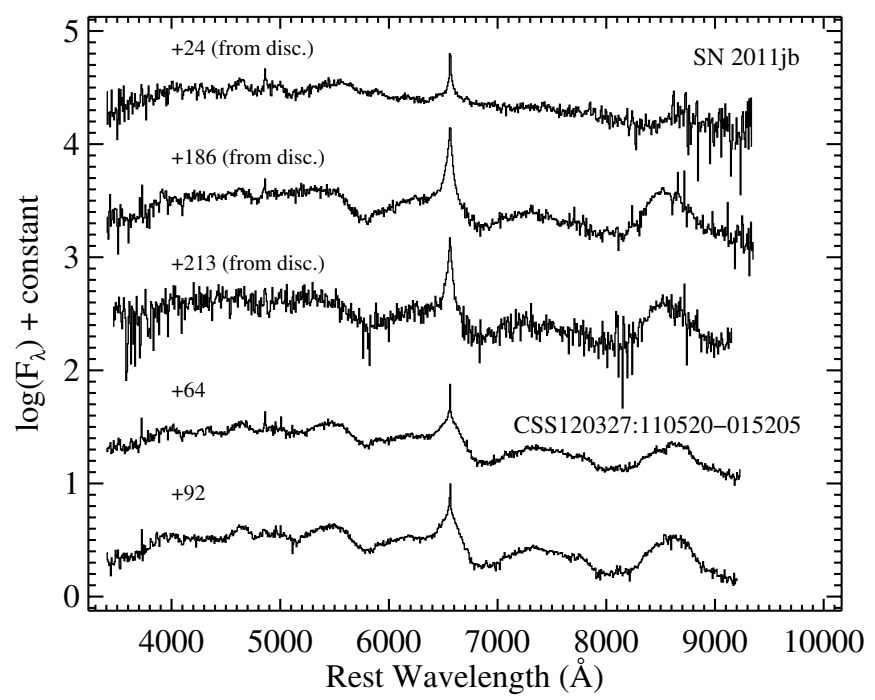

Figure 5. Spectra of SN 2011jb labeled with age relative to the discovery date and spectra of CSS120327:110520-015205 labeled with age relative to maximum brightness. The data have had their host-galaxy recession velocity removed and have been corrected for Galactic reddening.

\subsection{SN 2011jb and CSS120327:110520-015205}

Almost nothing has appeared in the literature regarding SNe 2011jb and CSS120327:110520-015205 (Drake et al. 2012), despite both objects being immediately classified as SNe Ia-CSM (Kankare et al. 2011; Drake et al. 2012). We present our three spectra of SN 2011jb and our two spectra of CSS120327:110520-015205 in Figure 5.

\section{PTF Ia-CSM OBJECTS}

A similar search for SNe Ia-CSM was performed using 178 spectra of all 63 SNe IIn discovered by PTF through 2012 August. Once again, we ran these spectra through SNID to see if they were well matched to our sample of SNe Ia-CSM. This initial analysis yielded nine possible objects, but two of these were rejected based on equally good spectral matches to $\mathrm{SNe}$ IIn. We classify the seven remaining objects as true SNe Ia-
Table 4

PTF SNe Ia-CSM

\begin{tabular}{lllcc}
\hline \hline SN Name & $\begin{array}{c}\text { Discovery } \\
\text { Date }\end{array}$ & $\begin{array}{c}\text { Approx. Date of } \\
r \text {-band Maximum }\end{array}$ & $\begin{array}{c}\text { Peak Absolute } \\
r \text {-band Magnitude }\end{array}$ & $\begin{array}{c}\text { Galactic } \\
\text { Reddening }\end{array}$ \\
\hline PTF11kx & 2011 Jan 16 & 2011 Jan 29 & -19.3 & 0.053 \\
\hline PTF10htz & 2010 Apr 3 & 2010 May 16 & -19.1 & 0.049 \\
PTF10iuf & 2010 Jun 5 & 2010 Jul 4 & -20.5 & 0.021 \\
PTF10yni & 2010 Oct 3 & 2010 Oct 30 & -20.6 & 0.059 \\
PTF11dsb & 2011 May 13 & $\ldots$ b & -19.8 & 0.020 \\
PTF11hzx & 2011 Jul 17 & 2011 Jul 23 & -21.3 & 0.096 \\
PTF12efc & 2012 May 13 & 2012 Jun 15 & -21.0 & 0.015 \\
PTF12hnr & 2012 Aug 7 & $\ldots$ b & -21.0 & 0.037
\end{tabular}

Notes.

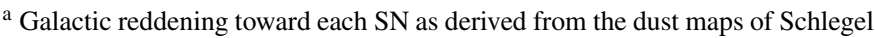
et al. (1998), including the corrections of Peek \& Graves (2010).

b $\mathrm{SN}$ was discovered after maximum brightness.

Table 5

PTF Ia-CSM Host Galaxies

\begin{tabular}{lccc}
\hline \hline SN Name & Name & Type & Redshift $z$ \\
\hline PTF11kx & SDSS J080913.20+461842.9 & Late-type spiral & 0.0466 \\
\hline PTF10htz & CGCG 352-058 & Late-type spiral & 0.0352 \\
PTF10iuf & SDSS J160615.65+335213.2 & Late-type spiral & 0.1586 \\
PTF10yni & $\ldots$ & $\ldots$ & 0.1688 \\
PTF11dsb & SDSS J161835.63+324150.0 & Late-type spiral & 0.1900 \\
PTF11hzx & $\ldots$ & $\ldots$ & 0.2287 \\
PTF12efc & $\ldots$ & $\ldots$ & 0.2341 \\
PTF12hnr & SDSS J232415.43-051239.0 & Late-type spiral & 0.1883
\end{tabular}

CSM. These PTF objects (in addition to PTF11kx) and their host galaxies are summarized in Tables 4 and 5, and our spectra of them are described in Table 6 and displayed in Figures 6-11. See Dilday et al. (2012) and Silverman et al. (2013) for further information regarding PTF11 kx and its spectra.

Three of the PTF SNe Ia-CSM were publicly announced while the other four were not. PTF10htz was initially classified as an SN IIb (Arcavi et al. 2010a), but our careful SNID analysis shows that it is likely an SN Ia-CSM (with substantial hostgalaxy contamination) and matches SNe 2011jb and 2005gj (Figure 6). In Arcavi et al. (2010b), PTF10yni (which was independently discovered by Drake et al. 2010, and called CSS101008:001049+141039) was classified as a "IIn/Ic," but once again our SNID analysis indicates that it is actually an SN Ia-CSM, matching SN 2005gj (Figure 8). Finally, PTF11dsb was classified simply as an "SN II" by Gal-Yam et al. (2011), but our analysis here shows that it is possibly an SN Ia-CSM, somewhat similar to SN 2011jb (Figure 8), though it is not completely clear whether this object is a bona fide member of the class.

\section{ANALYSIS AND DISCUSSION OF THE SN Ia-CSM CLASS}

Using our SNID analysis we have identified seven new SNe Ia-CSM from PTF (Section 3) and reidentified eight previously known SNe Ia-CSM (Section 2). Given these 15 objects, in addition to PTF11kx, we now attempt to define observational characteristics of this class of $\mathrm{SNe}$ Ia (while keeping in mind that PTF11kx may be an extreme member). Though no single observable appears to be a sufficient condition for an SN to be considered an Ia-CSM object, there seem to be a handful of features that nearly all of the SNe Ia-CSM display. 
Table 6

Spectra of PTF SNe Ia-CSM

\begin{tabular}{|c|c|c|c|c|c|}
\hline UT Date & $\begin{array}{l}\mathrm{Age}^{\mathrm{a}} \\
\text { (days) }\end{array}$ & Instrument $^{\mathrm{b}}$ & $\begin{array}{l}\text { Range } \\
(\AA)\end{array}$ & $\begin{array}{l}\text { Res. }^{c} \\
(\AA)\end{array}$ & $\begin{array}{c}\text { Exp. } \\
\text { (s) }\end{array}$ \\
\hline \multicolumn{6}{|c|}{ PTF10htz } \\
\hline 2010 Jun 13.3 & 27 & DBSP & $3500-9800$ & $3 / 4$ & 450 \\
\hline $2010 \mathrm{Jul} 14.2$ & 57 & DBSP & $3550-9900$ & $3 / 4$ & 450 \\
\hline \multicolumn{6}{|c|}{ PTF10iuf } \\
\hline 2010 Jun 7.1 & -23 & ISIS & $3150-9500$ & $3.5 / 7.2$ & 900 \\
\hline 2010 Jun 12.5 & -19 & LRIS & $3258-10116$ & $4.5 / 6.2$ & 900 \\
\hline $2010 \mathrm{Jul} 7.4$ & 3 & LRIS & $3300-10200$ & $4.5 / 6$ & 450 \\
\hline 2010 Aug 1.0 & 24 & ISIS & 3150-9500 & $3.5 / 7.2$ & 900 \\
\hline 2010 Aug 8.3 & 30 & DBSP & $3400-9935$ & $3 / 4$ & 750 \\
\hline 2010 Oct 12.2 & 86 & DEIMOS & $4480-9630$ & 3 & 750 \\
\hline \multicolumn{6}{|c|}{ PTF10yni } \\
\hline 2010 Nov 3.2 & 3 & RCS & $3330-8235$ & 5.9 & 1200 \\
\hline 2010 Dec 6.2 & 32 & DBSP & $3505-10000$ & $3 / 4$ & 600 \\
\hline 2010 Dec 13.2 & 38 & DBSP & 3500-9900 & $3 / 4$ & 1200 \\
\hline \multicolumn{6}{|c|}{ PTF11dsb } \\
\hline 2011 Jun 2.1 & 17 & LRIS & $3040-10240$ & $6.5 / 6$ & 450 \\
\hline $2011 \mathrm{Jul} 5.4$ & 45 & DEIMOS & $4592-7070$ & 3 & 600 \\
\hline \multicolumn{6}{|c|}{ PTF11hzx } \\
\hline $2011 \mathrm{Jul} 26.4$ & 3 & DBSP & $3440-9800$ & $3 / 4$ & 900 \\
\hline 2011 Aug 1.5 & 8 & DEIMOS & $4765-9633$ & 3 & 1000 \\
\hline 2011 Aug 6.4 & 12 & DBSP & $3400-9250$ & $3 / 4$ & 600 \\
\hline 2011 Aug 28.3 & 30 & DBSP & $3330-10000$ & $3 / 4$ & 430 \\
\hline 2011 Sep 29.4 & 56 & DEIMOS & $4583-8232$ & 3 & 1200 \\
\hline \multicolumn{6}{|c|}{ PTF12efc } \\
\hline 2012 May 17.5 & -23 & LRIS & $3382-10108$ & $3.7 / 5.9$ & 450 \\
\hline 2012 May 22.4 & -19 & LRIS & $3050-10257$ & $6.5 / 6$ & 480 \\
\hline 2012 May 29.3 & -14 & DBSP & $3480-10400$ & $3 / 4$ & 1200 \\
\hline 2012 Jun 18.3 & 2 & DBSP & $3388-10120$ & $3 / 4$ & 1800 \\
\hline $2012 \mathrm{Jul} 16.3$ & 25 & DEIMOS & $4500-8640$ & 3 & 800 \\
\hline \multicolumn{6}{|c|}{ PTF12hnr } \\
\hline 2012 Aug 9.5 & 2 & Kast & $3500-10000$ & $4 / 10$ & 2100 \\
\hline 2012 Aug 22.1 & 13 & ISIS & $3500-9476$ & $3.5 / 7.2$ & 1800 \\
\hline
\end{tabular}

Notes.

${ }^{a}$ Rest-frame days relative to maximum brightness, except for PTF11dsb and PTF12hnr where the epoch is relative to the date of discovery (2011 May 13 and 2012 August 7, respectively). See Table 4 for the dates of maximum brightness for the rest of the objects.

b DBSP: Double Spectrograph on the Palomar 200 inch telescope; ISIS: Intermediate dispersion Spectrograph and Imaging System on the $4.2 \mathrm{~m}$ William Herschel Telescope; LRIS: Low Resolution Imaging Spectrometer on the Keck $10 \mathrm{~m}$ telescope; DEIMOS: DEep Imaging Multi-Object Spectrograph on the Keck 10 m telescope; RCS: RC Spec on the KPNO 4 m telescope; Kast: Kast double spectrograph on the Shane $3 \mathrm{~m}$ telescope at Lick Observatory.

${ }^{c}$ Approximate FWHM resolution. If two numbers are listed, they represent the blue-side and red-side resolutions, respectively.

These are discussed in detail below, along with a few observables that are also found more generally in "normal" SNe IIn.

\subsection{Optical Photometry of SNe Ia-CSM}

The Caltech Core-Collapse Project (CCCP; Kiewe et al. 2012) found that the typical peak absolute magnitude range for SNe IIn is $-18.7 \mathrm{mag} \leqslant M_{R} \leqslant-17 \mathrm{mag}$ (using $H_{0}=$ $73 \mathrm{~km} \mathrm{~s}^{-1} \mathrm{Mpc}^{-1}$, the value adopted throughout this work), while the Lick Observatory SN Search (LOSS) found their $\mathrm{SNe}$ IIn to have peak luminosities in the range -19 mag $\leqslant$

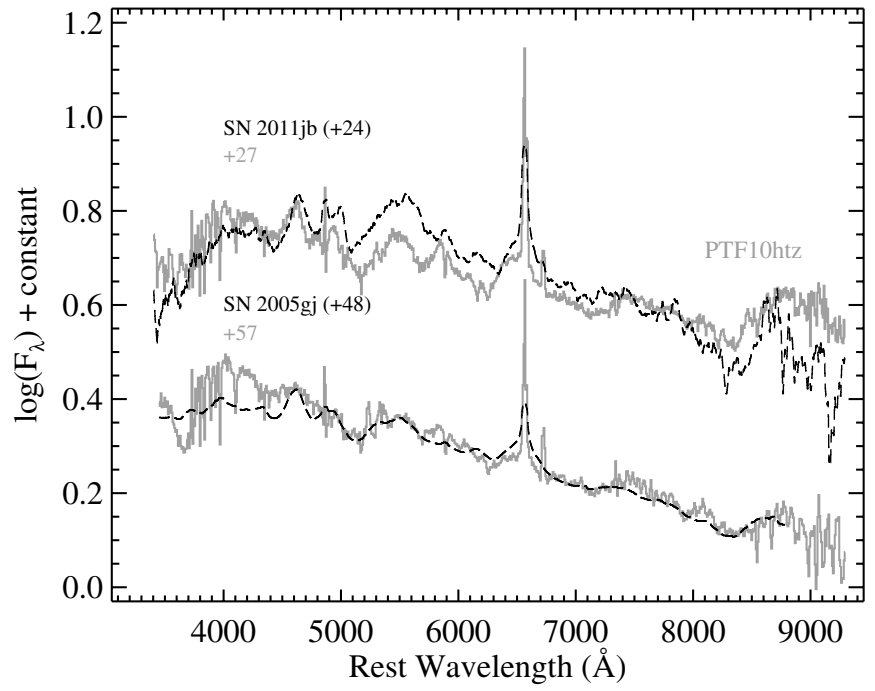

Figure 6. Spectra of PTF10htz labeled with age relative to maximum brightness, and comparison spectra (black). The data have had their host-galaxy recession velocity removed and have been corrected for Galactic reddening. Note that this object has a relatively large amount of host-galaxy contamination, but the superposed SN features match those of other SNe Ia-CSM quite well.

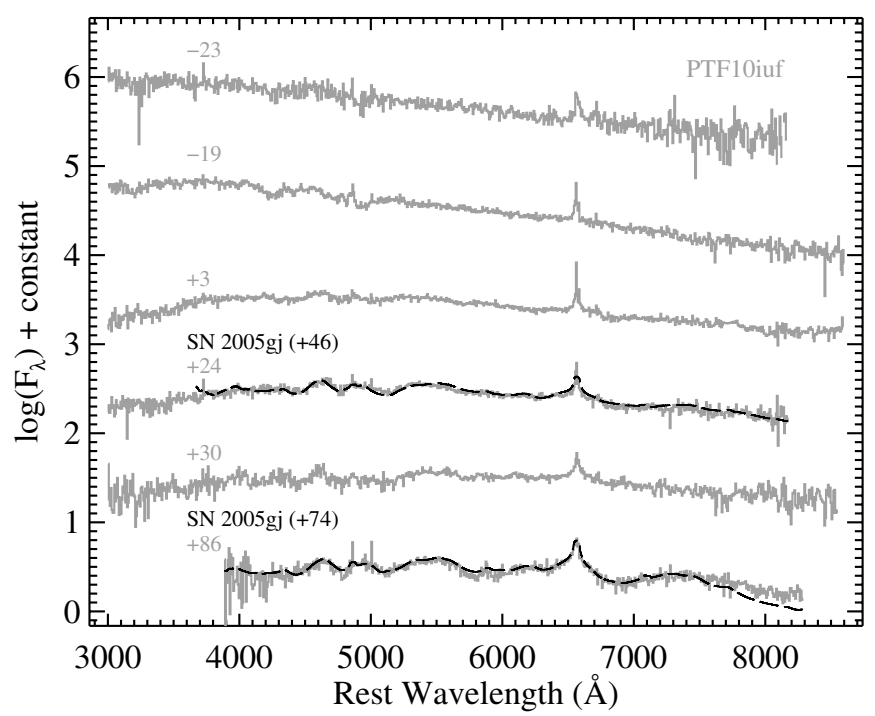

Figure 7. Spectra of PTF10iuf labeled with age relative to maximum brightness, and some comparison spectra (black). The data have had their host-galaxy recession velocity removed and have been corrected for Galactic reddening.

$M_{R} \leqslant-16 \mathrm{mag}$ (Li et al. 2011). LOSS also showed that SNe IIP/IIL have an overlapping range of peak absolute magnitudes ( $-17 \mathrm{mag} \leqslant M_{R} \leqslant-15 \mathrm{mag}$; Smith et al. 2011b), but the spectra of SNe IIP/IIL differ significantly from those of SNe IIn or SNe Ia-CSM (the former consisting of broad P-Cygni profiles of H Balmer lines; e.g., Filippenko 1997). On the less luminous end, luminous blue variable star (LBV) outbursts and the so-called SN impostors (which are spectroscopically similar to SNe IIn and SNe Ia-CSM) have peak absolute magnitudes $-16 \mathrm{mag} \leqslant M_{R}$ (Smith et al. 2011b). On the more luminous end, Type II superluminous $\mathrm{SNe}$ (which can also resemble SNe IIn and SNe Ia-CSM spectroscopically) have peak luminosities $M_{R} \lesssim-21 \mathrm{mag}$ (Gal-Yam 2012; Quimby et al. 2013). We note that both CCCP and LOSS have a dearth of objects which are spectroscopically similar to SNe IIn with peak absolute magnitudes $-21 \mathrm{mag} \leqslant M_{R} \leqslant-19 \mathrm{mag}$, although the PTF sample contains quite a few objects in this range (Figure 12). 


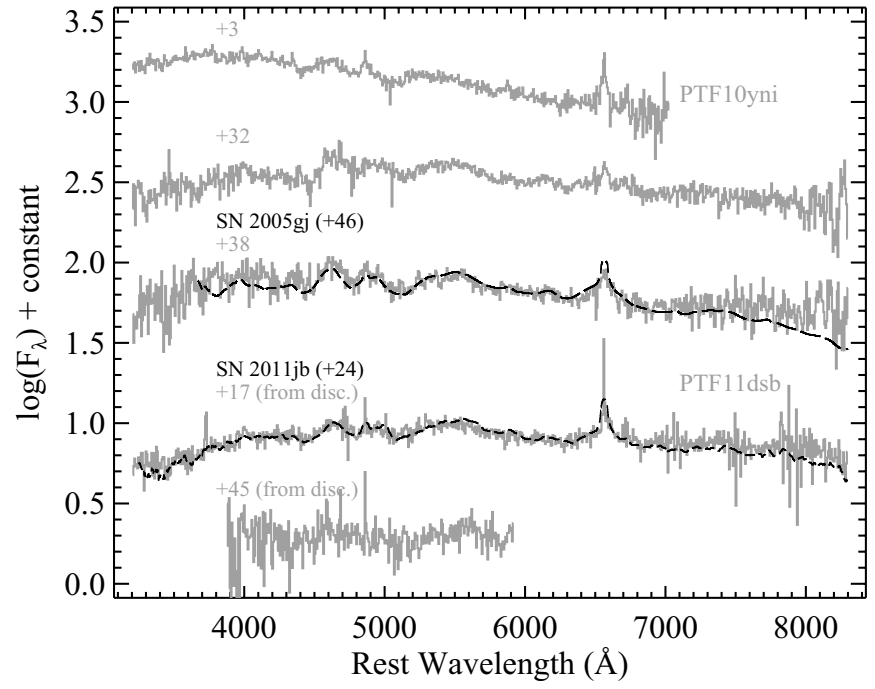

Figure 8. Spectra of PTF10yni labeled with age relative to maximum brightness and spectra of PTF11dsb labeled with age relative to the discovery date, and some comparison spectra (black). The data have had their host-galaxy recession velocity removed and have been corrected for Galactic reddening.

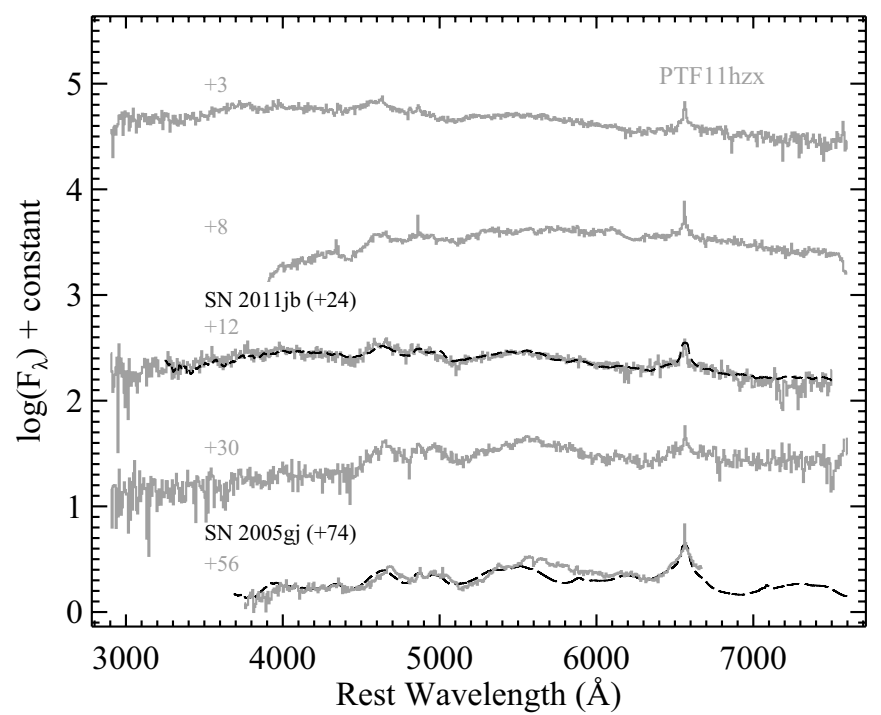

Figure 9. Spectra of PTF11hzx labeled with age relative to maximum brightness, and some comparison spectra (black). The data have had their host-galaxy recession velocity removed and have been corrected for Galactic reddening.

Extending this analysis, in Figure 12 we plot the peak absolute $r$-band magnitude of all 63 objects spectroscopically identified as SNe IIn by PTF through 2012 August. The photometric calibration of the PTF data is based on the Sloan Digital Sky Survey (SDSS) data when possible, otherwise the PTF calibration and natural magnitude system are used (Ofek et al. 2012a, 2012b). The vertical dotted lines denote the boundaries between the various subtypes of SNe IIn (LBV outbursts and SN impostors, typical SNe IIn, and superluminous SNe II) and our proposed range of SN Ia-CSM luminosities. The gray shaded region is the range of SNe Ia that follow the Phillips relation, about -19.7 to $-18.5 \mathrm{mag}$ (e.g., Ganeshalingam et al. 2010). The black, filled histogram shows the peak absolute magnitudes of the seven SNe Ia-CSM discovered by PTF (Section 3) not including PTF11kx, the downward-pointing arrows signify the peak luminosities of the eight previously known SNe Ia-CSM

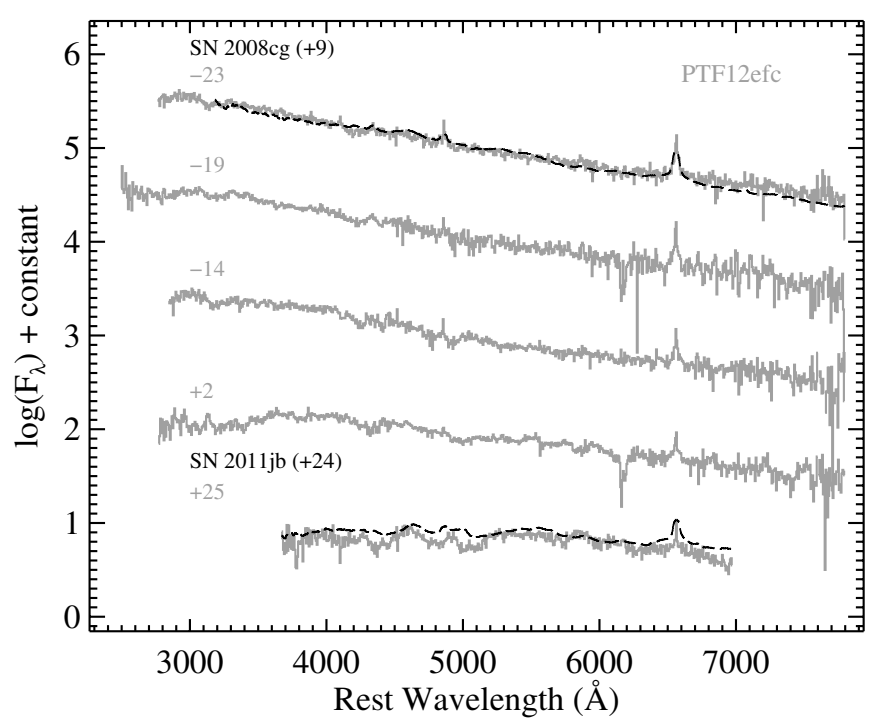

Figure 10. Spectra of PTF12efc labeled with age relative to maximum brightness, and some comparison spectra (black). The data have had their host-galaxy recession velocity removed and have been corrected for Galactic reddening.

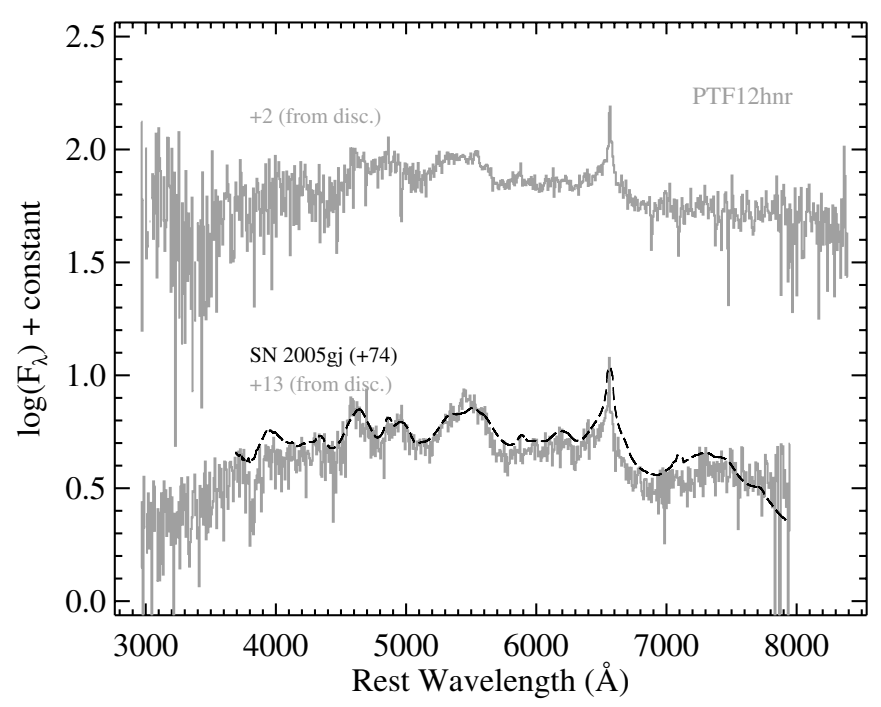

Figure 11. Spectra of PTF12hnr labeled with age relative to the discovery date, and some comparison spectra (black). The data have had their host-galaxy recession velocity removed and have been corrected for Galactic reddening.

(Section 2), and the star represents the peak absolute magnitude of PTF11kx (Dilday et al. 2012).

As seen in Figure 12, 15 of the $16 \mathrm{SNe}$ Ia-CSM discussed in this paper fall in the luminosity range $-21 \mathrm{mag} \leqslant M_{R} \leqslant$ -19 mag. The one outlier, PTF11hzx, just barely misses this cutoff with peak luminosity $M_{R}=-21.3$ mag. It appears that SNe Ia-CSM must have an absolute optical magnitude in this range, roughly $0.5-1.5 \mathrm{mag}$ brighter than their more normal SN Ia cousins, likely due to the interaction of the SN ejecta with the CSM (which gives SNe Ia-CSM their distinct spectra).

We also investigate the rise times of SN Ia-CSM light curves (i.e., the time elapsed between explosion and maximum brightness). Ganeshalingam et al. (2011) found that normal SNe Ia have a rise time of $\sim 18$ days in the $B$ band and $\sim 20$ days in the $V$ band (after correcting for light-curve shape). Longer rise times have been seen in more exotic SN Ia subtypes, with 




Figure 12. Peak absolute $r$-band magnitude of all 63 SNe IIn discovered by PTF through 2012 August. The vertical dotted lines denote the boundaries between the various subtypes of SNe IIn and SNe Ia-CSM, and the gray shaded region is the range of SNe Ia that follow the Phillips relation. The black, filled histogram shows the seven SNe Ia-CSM discovered by PTF (not including PTF11kx), the downward-pointing arrows are the eight previously known SNe Ia-CSM, and the star is PTF11kx.

possible super-Chandrasekhar mass SNe Ia having rise times of 24 days (e.g., Scalzo et al. 2010; Silverman et al. 2011).

Accurate rise-time measurements require photometric observations well before maximum brightness; thus, not many of the previously studied SNe Ia-CSM have reasonable rise-time constraints. SN 2002ic was found to have a rise time as long as 28 days (Wood-Vasey et al. 2004), while SN 2005gj had a rise time of $\sim 20$ days in the $g$ band and possibly up to $\sim 32$ days in the $r$ band (Aldering et al. 2006; Prieto et al. 2007). On the other hand, CSS120327:110520-015205 appeared to have a rise time of $\sim 45$ days (Drake et al. 2012). As for the PTF SNe Ia-CSM, Dilday et al. (2012) used a rise time of $\sim 20$ days for PTF11kx. Five of the seven other SNe Ia-CSM from PTF have constraining pre-maximum brightness photometry and show evidence for relatively long rise times ( $\sim 30-40$ days).

Thus, it appears that SNe Ia-CSM tend to have longer rise times than more normal SNe Ia. This is consistent with the idea that the rise time is related to the photon diffusion time (which should be longer in SNe Ia-CSM as the light must make its way out of the relatively large amount of CSM) as well as models of circumstellar shells in symbiotic recurrent nova systems (Moore $\&$ Bildsten 2012). These rise times can also be used to estimate the mass-loss rate of the progenitor system that gave rise to the CSM. Using typical SN Ia-CSM ejecta and wind velocities of a few thousand and $100 \mathrm{~km} \mathrm{~s}^{-1}$, respectively, and equations in Ofek et al. (2013), we find that SNe Ia-CSM have mass-loss rates of a few times $10^{-1} M_{\odot} \mathrm{yr}^{-1}$.

\subsection{Optical Spectroscopy of SNe Ia-CSM}

We identified SNe Ia-CSM using our SNID analysis outlined in Sections 2 and 3. These matches were based solely on comparing low-resolution optical spectra of input $\mathrm{SNe}$ with a library of template spectra. When comparing the spectra of the SNe Ia-CSM, some show relatively strong underlying SN Ia features (e.g., SNe 2002ic and 2005gj, and especially PTF11kx), mostly at early times and often resembling the somewhat overluminous Type Ia SN 1999aa (Li et al. 2001; Strolger et al. 2002; Garavini et al. 2004). However, other

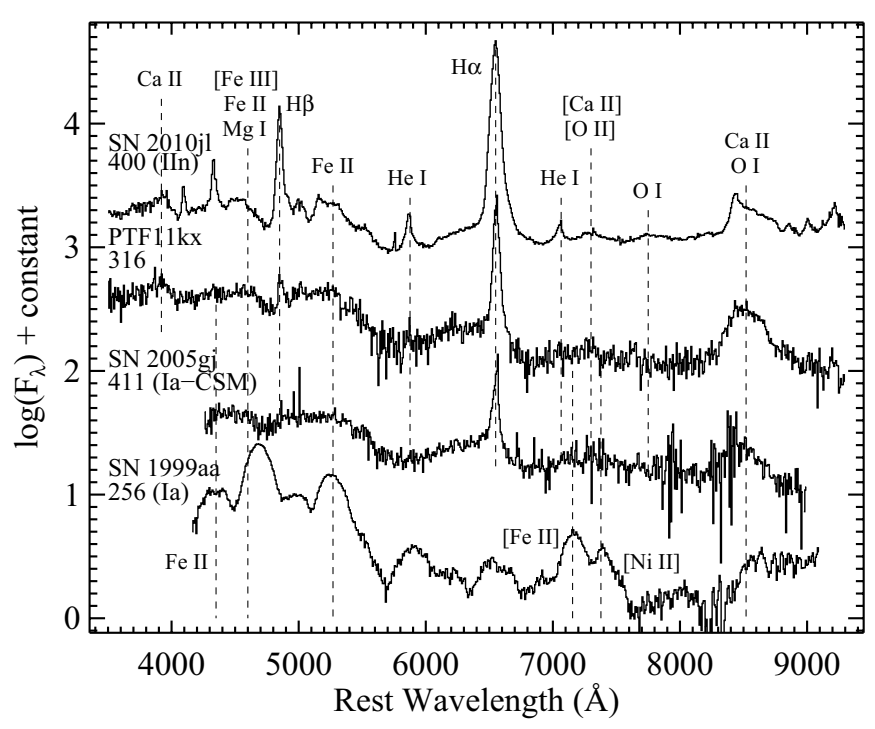

Figure 13. Spectra of PTF11kx and SN 2005gj, in addition to SN 2010j1 (an SN IIn; Smith et al. 2011a) and SN 1999aa (a somewhat overluminous SN Ia; Silverman et al. 2012a). Each spectrum is labeled with its rest-frame age relative to maximum brightness and major spectral features are labeled. The data have had their host-galaxy recession velocity removed and have been corrected for Galactic reddening. The figure is reproduced from Silverman et al. (2013).

SNe Ia-CSM (mostly ones with spectra from later epochs) do not resemble any subtype of SN Ia, aside from the other SNe IaCSM, and are much more easily mistaken for more typical SNe IIn.

At these late times, the spectra of SNe Ia-CSM basically consist of a relatively blue "quasi-continuum," which is likely due to emission from many overlapping, relatively narrow lines of iron-group elements (IGEs), mostly Fe II, excited by the CSM interaction, in addition to strong $\mathrm{H} \alpha$ emission and often broad Ca II emission (e.g., Deng et al. 2004). Figure 13 shows spectra of PTF11kx and SN 2005gj, along with two comparison objects: SN 2010j1 (an SN IIn; Smith et al. 2011a) and SN 1999aa (a somewhat overluminous SN Ia; Silverman et al. 2012a). The figure is reproduced from Silverman et al. (2013) and some of the major spectral features are labeled.

We do not find strong evidence of oxygen in SNe Ia-CSM, which is often prominent in SNe IIn in the form of the O I $\lambda 7774$ feature. However, it is possible that part of the very broad emission feature around $7400 \AA$ (see especially Figures 1-5) is produced by $\mathrm{O}_{\mathrm{I}} \lambda 7774$ blended with $\left[\mathrm{O}_{\mathrm{II}}\right] \lambda \lambda 7319,7330$, along with the almost certain [Ca II] $\lambda \lambda 7291,7324$. Moreover, note that the broad emission just blueward of $\mathrm{H} \alpha$ often seen in SNe Ia-CSM might be due to [O I] $\lambda \lambda 6300,6364$, adding to the evidence for oxygen emission. However, it could more likely be related to the broad $\mathrm{H} \alpha$ emission underlying the narrower components discussed at length below.

To further investigate the spectral characteristics of SNe IaCSM (specifically as compared to SNe IIn), we measure properties of the $\mathrm{H} \alpha, \mathrm{H} \beta$, and $\mathrm{He}$ I $\lambda 5876$ features in all of the objects classified as SNe IIn from the Berkeley SN Group's database (Silverman et al. 2012a) as well as the 63 SNe IIn discovered by PTF through 2012 August. We follow the procedure of Dilday et al. (2012) and fit the spectral features with Gaussian profiles, using multiple components when necessary. In the following discussion, we are referring to the broader component with FWHM of about 500-2000 $\mathrm{km} \mathrm{s}^{-1}$, unless otherwise specified. Furthermore, we found that the similarities and differences between SNe IIn and SNe Ia-CSM were relatively persistent at 
all epochs, and thus in the analysis below we consider data on all SNe IIn and SNe Ia-CSM at all epochs.

We find that SNe Ia-CSM tend to have $\mathrm{H} \alpha$ profiles which, for the most part, match the bulk of the SN IIn distribution. The velocity associated with the peak of the $\mathrm{H} \alpha$ emission in SNe Ia-CSM is slightly redshifted from the systemic velocity of their host galaxies, whereas the SN IIn peaks match their hostgalaxy velocities more closely. However, this redshift is not statistically significant. Also, the equivalent width (EW) and FWHM of $\mathrm{H} \alpha$ are slightly smaller in SNe Ia-CSM as compared with SNe IIn, but again this is not a statistically significant result. For SNe Ia-CSM having multiple spectra, we find that the EW of $\mathrm{H} \alpha$ shows strong fluctuations until 100-150 days past maximum brightness, perhaps indicating the $\mathrm{SN}$ ejecta are interacting with multiple CSM shells of varying sizes and/or densities. At later epochs, the EW tends to increase with time until $\sim 1$ yr past maximum, when the EW possibly begins to decrease. This overall behavior has been seen previously in PTF11kx (Dilday et al. 2012; Silverman et al. 2013), SN 2002ic (Wang et al. 2004), and SN 2005gj (Aldering et al. 2006; Prieto et al. 2007).

The typical luminosities of $\mathrm{H} \alpha$ emission in nearly all of the SNe Ia-CSM studied herein (including PTF11kx) fall in the range (1-9) $\times 10^{40} \mathrm{erg} \mathrm{s}^{-1}$. The only object that lies outside this range is CSS120327:110520-015205 (which has a luminosity of $\sim 3.9 \times 10^{41} \mathrm{erg} \mathrm{s}^{-1}$ ). Previously calculated $\mathrm{H} \alpha$ luminosities of $\mathrm{SNe}$ Ia-CSM are consistent with this range. Specifically, SN 2002ic had a luminosity of $\sim 5 \times 10^{40} \mathrm{erg} \mathrm{s}^{-1}$ (Kotak et al. 2004), SN 2005gj had luminosities of (1-10) $\times 10^{40} \mathrm{erg} \mathrm{s}^{-1}$ (Aldering et al. 2006; Prieto et al. 2007), and SN 2008J had a luminosity of $\sim 1.3 \times 10^{41} \mathrm{erg} \mathrm{s}^{-1}$ (Taddia et al. 2012).

In their respective references (and assuming shock velocities of a few thousand $\mathrm{km} \mathrm{s}^{-1}$ and wind velocities of $\sim 100 \mathrm{~km} \mathrm{~s}^{-1}$ ), the $\mathrm{H} \alpha$ luminosities of these three SNe Ia-CSM were converted into mass-loss rates in the range $(2-120) \times 10^{-4} M_{\odot} \mathrm{yr}^{-1}$, which is quite a bit lower than the mass-loss rates we infer using the rise times of SNe Ia-CSM. This may be due to the fact that these estimates usually assume that the $\mathrm{H} \alpha$ emission is produced by optically thin CSM being ionized by the $\mathrm{SN}$ radiation field, whereas we will show below that the $\mathrm{H} \alpha$ feature is instead likely produced largely by collisional excitation.

In more typical SNe IIn (i.e., ones that almost certainly came from the core collapse of a massive star), decreased flux in the red wing of $\mathrm{H} \alpha$ compared with the blue wing has been interpreted as a sign of new dust forming in the post-shock material (e.g., Fox et al. 2011; Smith et al. 2012). In fact, Fox et al. (2011) showed this exact phenomenon for SNe 2008J and $2008 \mathrm{cg}$. Figures $14-16$ display the $\mathrm{H} \alpha$ profiles of the $12 \mathrm{SNe}$ IaCSM for which we present spectra in this paper. Following the method of Fox et al. (2011), we first remove a linear continuum near $\mathrm{H} \alpha$ from each spectrum (the long-dashed lines in the figures represent the now-horizontal continuum level). Next we reflect the blue half of the $\mathrm{H} \alpha$ profile across the peak flux, yielding the short-dashed lines in the figures. The dotted vertical lines are the systemic velocity of each object.

We recover the result of Fox et al. (2011) that SNe 2008J and $2008 \mathrm{cg}$ both show diminished flux in the red wing of $\mathrm{H} \alpha$ compared with the blue wing. Surprisingly, we see the exactly same behavior in nearly all of the other non-PTF SNe Ia-CSM at similar epochs. The red wings begin to decrease in flux at 75-100 days past maximum brightness and seem to continue decreasing with time. We do note, however, that there is some evidence that the red flux in SN 2008J begins to increase again at the latest epochs covered by our spectra. We do not see this behavior in the PTF SNe Ia-CSM because we lack spectra at epochs later than $\sim 75$ days past maximum brightness (except for one spectrum of PTF10iuf taken 86 days past maximum, which possibly shows evidence of a slight decrease in flux in the red wing of $\mathrm{H} \alpha$, as does the spectrum of PTF11hzx taken 55 days past maximum). In Fox et al. (2011), $10^{-3}-10^{-2} M_{\odot}$ of dust was inferred from the mid-IR observations of SNe 2008J and $2008 \mathrm{cg}$, but we caution that there are quite a few assumptions going into the conversion from mid-IR photometry to dust mass. Interestingly, PTF11kx does not show this evidence for dust formation through 436 days past maximum brightness. However, there is some evidence of a decrease in the red wing of $\mathrm{H} \alpha$ in the spectrum from 680 days past maximum (Silverman et al. 2013).

In contrast to $\mathrm{H} \alpha$, the $\mathrm{EW}$ of $\mathrm{H} \beta$ in SNe Ia-CSM is significantly smaller than that of normal SNe IIn. The median $\mathrm{H} \beta$ EW for SNe Ia-CSM is $\sim 6 \AA$, yet it is $\sim 13 \AA$ for SNe IIn. Furthermore, the EW values of the two $\mathrm{SN}$ types seem to be drawn from different parent populations; a Kolmogorov-Smirnov (KS) test yields $p \approx 0.0003$. The top panel of Figure 17 shows the cumulative fraction of $\mathrm{H} \beta \mathrm{EW}$ s for the SNe IIn from the Berkeley SN Group's database as well as the SNe IIn from PTF. The $\mathrm{SNe}$ Ia-CSM with spectra presented in this work are represented by the gray line while the SNe IIn are represented as the black line. The median EW was used when there were multiple EW measurements for a given object.

Similarly, we can calculate the $\mathrm{H} \alpha / \mathrm{H} \beta$ intensity ratio (hereafter, the Balmer decrement) of the SNe IIn and SNe Ia-CSM, which is shown in the bottom panel of Figure 17. The Balmer decrement is only somewhat smaller in SNe IIn compared with $\mathrm{SNe}$ Ia-CSM ( $\sim 3$ versus $\sim 5$, respectively), but according to a $\mathrm{KS}$ test this is significant ( $p \approx 0.024)$. The Balmer decrement in SNe Ia-CSM also appears to increase with time before eventually decreasing, peaking at ages anywhere from about a few months to a year and a half past maximum brightness. PTF11kx had $\mathrm{H} \alpha / \mathrm{H} \beta>7$ in all of its late-time spectra (Silverman et al. 2013), and large Balmer decrements were also observed in SN 2005gj (Aldering et al. 2006).

A possible explanation for these large Balmer decrements is that the emission lines are produced primarily through collisional excitation rather than recombination. Recombination should lead to a Balmer decrement of $\sim 3$, while moderately high-density gas can lead to large Balmer decrements when the optical depth of $\mathrm{H} \alpha$ is large (Drake \& Ulrich 1980), possibly caused by Balmer self-absorption and collisional excitation (Xu et al. 1992). In SNe Ia-CSM, the SN ejecta may be interacting with thin, relatively dense, slowly moving shells of CSM that have cavities on either side of the shell, as one would expect from recurrent nova eruptions (and as was suggested for PTF11kx; Dilday et al. 2012). When the rapidly moving SN ejecta catch up to and overtake the more slowly moving thin, dense shells, the hydrogen may get collisionally excited and naturally lead to the large $\mathrm{H} \alpha / \mathrm{H} \beta$ ratios observed in the spectra of SNe Ia-CSM. Furthermore, models of SNe IaCSM interacting with a wind having a constant mass-loss rate appear to be inconsistent with late-time photometric observations (Chugai \& Yungelson 2004). Thus, this relatively large Balmer decrement, which is ubiquitous in SNe Ia-CSM, is likely caused by interaction with multiple thin, dense shells of CSM.

The EW of He I $\lambda 5876$ was relatively small in PTF $11 \mathrm{kx}$ ( 9 Aं; Dilday et al. 2012; Silverman et al. 2013), smaller than 

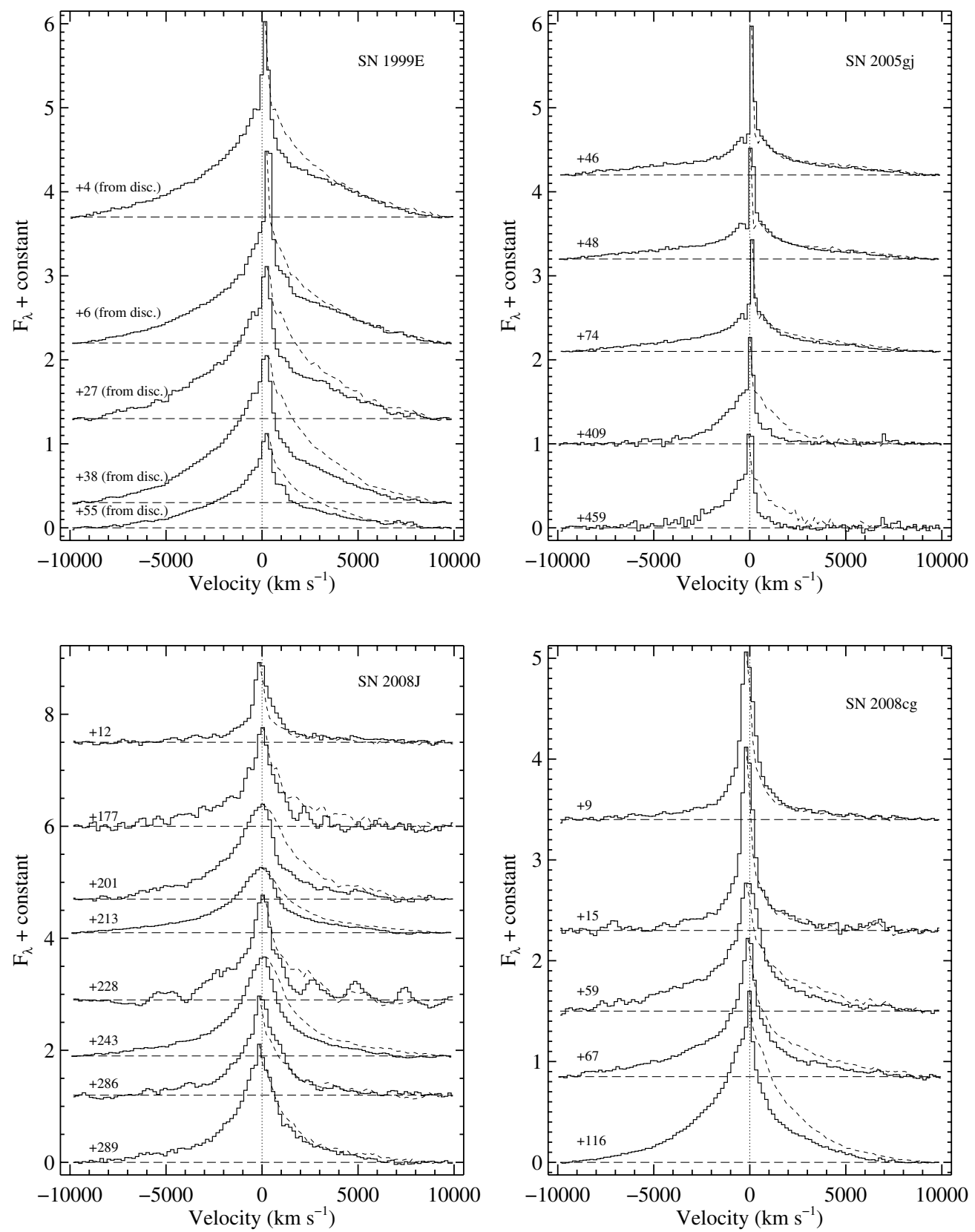

Figure 14. $\mathrm{H} \alpha$ profiles of SNe 1999E, 2005gj, 2008J, and 2008cg. After removing a linear continuum (the long-dashed lines represent the now-horizontal continuum level), we reflect the blue half of the $\mathrm{H} \alpha$ profile across the peak flux, yielding the short-dashed lines. The dotted vertical line is the systemic velocity of each object.

that of most normal SNe IIn. Weak He I emission extends beyond just PTF11kx to the rest of the SNe Ia-CSM as well. Relatively narrow He I emission was detected in optical and near-IR spectra of SN 2008J taken a few days before maximum brightness, but the features were weak (Taddia et al. 2012). Figure 18 shows the cumulative fraction of EW of He I $\lambda 5876$ for the Berkeley Group's SNe IIn and the PTF SNe IIn in black, and for SNe IaCSM that have spectra shown in this work in red. The blue line is the $\mathrm{SNe}$ Ia-CSM, but including upper limits to the $\mathrm{He}$ I $\lambda 5876$ $\mathrm{EW}$, and the green line includes the upper limits for the nonSNe Ia-CSM. As above, the median EW is used when multiple EW measurements exist for a given object.

A KS test shows that the difference between SNe Ia-CSM and $\mathrm{SNe}$ IIn is significant $(p \approx 0.009)$, and the difference in median EW is striking ( $\sim 2 \AA$ versus $\sim 6 \AA$, respectively).
Including the upper limits calculated for the $\mathrm{EW}$ of $\mathrm{He}$ I $\lambda 5876$, the difference is decreased (median EW values of $\sim 2$ and $\sim 4 \AA$ for SNe Ia-CSM and SNe IIn, respectively), and it is less significant $(p \approx 0.017)$. Thus, it seems that little to no He I emission is a common attribute of members of the SN Ia-CSM class. Perhaps the stronger He I emission in SNe IIn is due to an actual abundance enhancement in the wind from a massive star, as compared to the CSM shells coming from systems containing a WD (e.g., Chevalier \& Fransson 1994).

As a final note on optical spectroscopy of SNe Ia-CSM, we find that in most objects and at nearly all epochs the second strongest feature (after $\mathrm{H} \alpha)$ is broad $\left(\sim 10,000 \mathrm{~km} \mathrm{~s}^{-1}\right)$ emission from the Ca II near-IR triplet. Every SN Ia-CSM discussed herein (with spectra that encompass this spectral feature near $8500-8600 \AA$ ) shows strong emission from the 

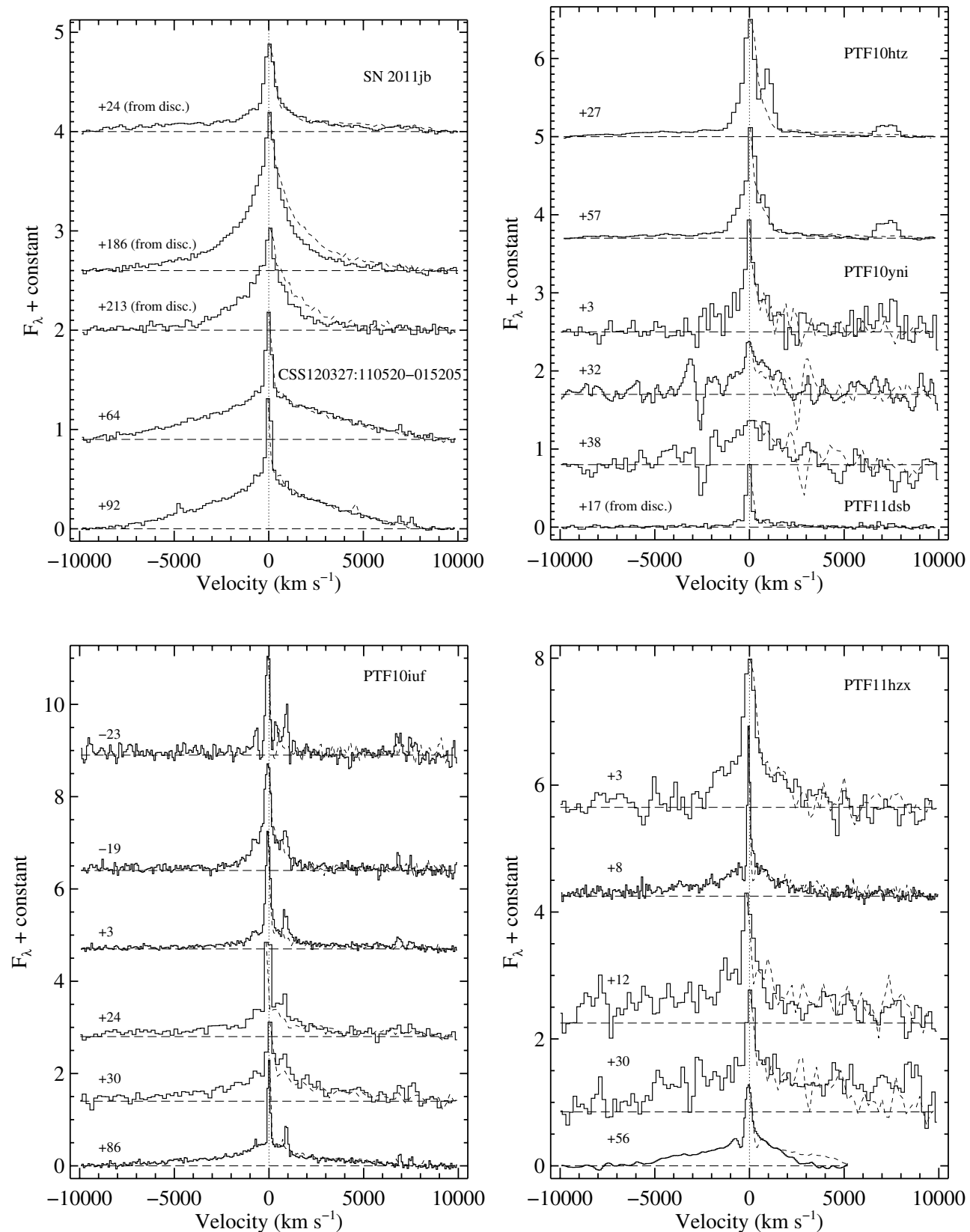

Figure 15. H $\alpha$ profiles of SN 2011jb, CSS120327:110520-015205, PTF10htz, PTF10yni, PTF10iuf, and PTF11hzx. After removing a linear continuum (the longdashed lines represent the now-horizontal continuum level), we reflect the blue half of the $\mathrm{H} \alpha$ profile across the peak flux, yielding the short-dashed lines. The dotted vertical line is the systemic velocity of each object.

Ca II near-IR triplet (see Figures 1-11). The feature also appears prominently in late-time spectra of PTF11kx, though it almost completely disappears by 680 days past maximum brightness (Silverman et al. 2013). According to at least one model, this behavior may be the result of a cool, dense shell with separate Fe-poor and Fe-rich zones becoming fully mixed (Chugai et al. 2004).

\subsection{Other Observations of SNe Ia-CSM}

In addition to optical photometry and low-resolution optical spectroscopy, there has been a smattering of other types of observations of SNe Ia-CSM at a variety of wavelengths. High-resolution optical spectra exist of relatively few $\mathrm{SNe}$, and this is true of SNe Ia-CSM as well. However, for all $\mathrm{SNe}$ Ia-CSM with published high-resolution spectroscopy, narrow (50-100 $\mathrm{km} \mathrm{s}^{-1}$ ) P-Cygni profiles have been observed in $\mathrm{H} \alpha$, as well as a handful of other spectral features (e.g., Kotak et al. 2004; Aldering et al. 2006; Dilday et al. 2012). Unfortunately, the spectra of SNe Ia-CSM presented herein are of such low resolution that we do not expect to observe these subtle features.

Weak, narrow Na I D absorption from the host galaxy of SN 2005gj was found in the high-resolution optical spectra presented by Aldering et al. (2006). On the other hand, Dilday et al. (2012) show strong Na I D absorption from the host galaxy of PTF11kx. Furthermore, a statistical study of many high-resolution optical spectra of $\mathrm{SNe}$ was undertaken by Sternberg et al. (2011), and the two SNe Ia-CSM in their sample (SNe 2008J and $2008 \mathrm{cg}$, referred to as CCSNe) are found to have saturated $\mathrm{Na}$ I $\mathrm{D}$ lines, though the lines were redshifted in SN 2008J and blueshifted in SN 2008cg. 

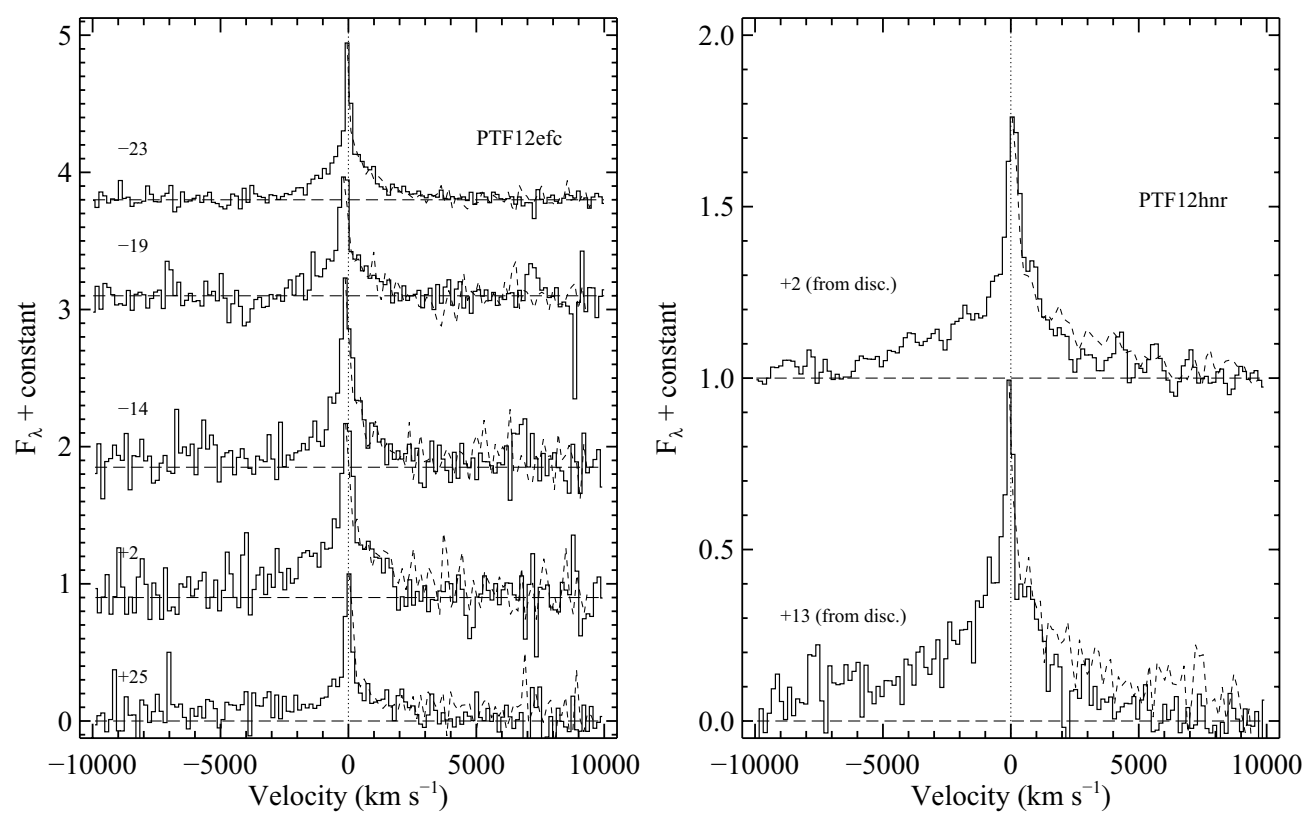

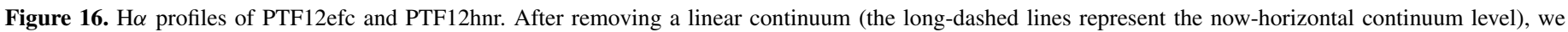
reflect the blue half of the $\mathrm{H} \alpha$ profile across the peak flux, yielding the short-dashed lines. The dotted vertical line is the systemic velocity of each object.

We inspect the low-resolution spectra presented in this work and find that all SNe Ia-CSM have likely Na I D absorption from the host galaxy. Regarding the two SNe Ia-CSM for which we do not present spectra herein, SN 1997cy probably does not show Na I D absorption from the host (Turatto et al. 2000; Germany et al. 2000), while SN 2002ic likely does (Benetti et al. 2006). Thus, narrow Na I D absorption from the host galaxy is present in nearly all SNe Ia-CSM.

High-resolution spectroscopy of $\mathrm{SNe}$ may be rare, but SN 2002ic is the only Ia-CSM object that has published spectropolarimetric observations. Wang et al. (2004) find that this object is mostly evenly polarized across the optical region at the $\sim 0.8 \%$ level, except near $\mathrm{H} \alpha$ where it is highly depolarized. They state that this is significantly different than more normal SNe Ia, and that in the case of SN 2002ic the polarization is likely more closely tied to the CSM than to the explosion itself.

At longer wavelengths than optical, we have already pointed out (in Section 2.3) that SNe 2008J and 2008cg are detected in the mid-IR by Fox et al. (2011). In fact, at $\sim 620$ days past maximum brightness, SN 2008J is the second strongest IR detection in their study, with flux densities of $2.52 \mathrm{mJy}$ and $2.71 \mathrm{mJy}$ at $3.6 \mu \mathrm{m}$ and $4.5 \mu \mathrm{m}$, respectively. While not as luminous, $\mathrm{SN} 2008 \mathrm{cg}$ at $\sim 530$ days past maximum is also clearly detected with flux densities of $0.30 \mathrm{mJy}$ and $0.35 \mathrm{mJy}$ at $3.6 \mu \mathrm{m}$ and $4.5 \mu \mathrm{m}$, respectively. The two best-studied SNe IaCSM are also found to have IR excesses at late times. SN 2002ic is easily detected in $K$-band imaging $\sim 256$ days past maximum brightness as well as in $H$-band and $K$-band imaging $~ 352$ days past maximum (Kotak et al. 2004). From 40-140 days past maximum, SN 2005gj is found to be 2-3 mag brighter and to decline more slowly than normal SNe Ia and SNe IIn in $J H K_{S}$ imaging (Prieto et al. 2007). These IR excesses are consistent with the new dust formation scenario described above in order to explain the decreasing flux in the red wing of $\mathrm{H} \alpha$ at late times. In addition, there exists only one published near-IR spectrum (extending to $2.2 \mu \mathrm{m}$ ) of an Ia-CSM object (SN 2008J), and it shows emission lines of the H Paschen and Brackett series, along with likely Fe II emission and weak He I emission (Taddia et al. 2012).

Proceeding to even lower energy radiation, SN 1997cy is not detected at 13 or $20 \mathrm{~cm} \sim 400$ days after discovery, though the limits $\left(\sim 2 \times 10^{21} \mathrm{~W} \mathrm{~Hz}^{-1}\right)$ are not very restrictive (Germany et al. 2000). Similarly, neither SN 2005gj (at 39 days past maximum brightness; Soderberg \& Frail 2005) nor SN 2008cg (at $\sim 61$ days past maximum; Chandra \& Soderberg 2008) is detected at $8.46 \mathrm{GHz}$ with the Very Large Array. PTF12hnr, observed at $6.1 \mathrm{GHz}$ using the EVLA $\sim 20$ days after discovery, also resulted in a null detection. These observations can be converted into radio luminosity upper limits of $\sim 10^{26}-10^{28} \mathrm{erg} \mathrm{s}^{-1} \mathrm{~Hz}^{-1}$. This range encompasses both upper limits and actual detections of more typical SNe IIn (e.g., Germany et al. 2000; Pooley et al. 2002; Fox et al. 2011).

At higher energies, both SN 2005gj (Immler et al. 2005) and SN 2008cg (Immler et al. 2008) are easily detected $\sim 39$ and $\sim 62$ days past maximum, respectively, by Swift/UVOT in the ultraviolet, including the bluest filter which covers 112-264 nm. PTF11hzx and PTF12efc, also observed by Swift/UVOT (the former $\sim 9$ days after maximum brightness and the latter $\sim 29$ and 16 days before maximum), are both well detected in all observations.

Moving into the X-rays, SN 2005gj was not detected by Swift/XRT $\sim 39$ days past maximum brightness (Immler et al. 2005) or by Chandra/ACIS $\sim 55$ days past maximum (Prieto et al. 2007). Similarly, no detection of SN 2008cg was made by Swift/XRT $\sim 62$ days past maximum brightness (Immler et al. 2008). Unsurprisingly, PTF11hzx was also not detected by Swift/XRT $\sim 9$ days after maximum brightness. The upper limits on the X-ray luminosity implied by these null detections are $\sim 10^{39}-10^{43} \mathrm{erg} \mathrm{s}^{-1}$; observed X-ray fluxes of SNe IIn fall in this range (e.g., Pooley et al. 2002; Zampieri et al. 2005; Immler et al. 2007). Using the upper limits determined from the X-ray nondetections of SNe Ia-CSM and equations in Ofek et al. (2013), we calculate mass-loss rate upper limits of a few times $10^{-1} M_{\odot} \mathrm{yr}^{-1}$ (which matches the mass-loss rates calculated above using the rise times of SNe Ia-CSM). 

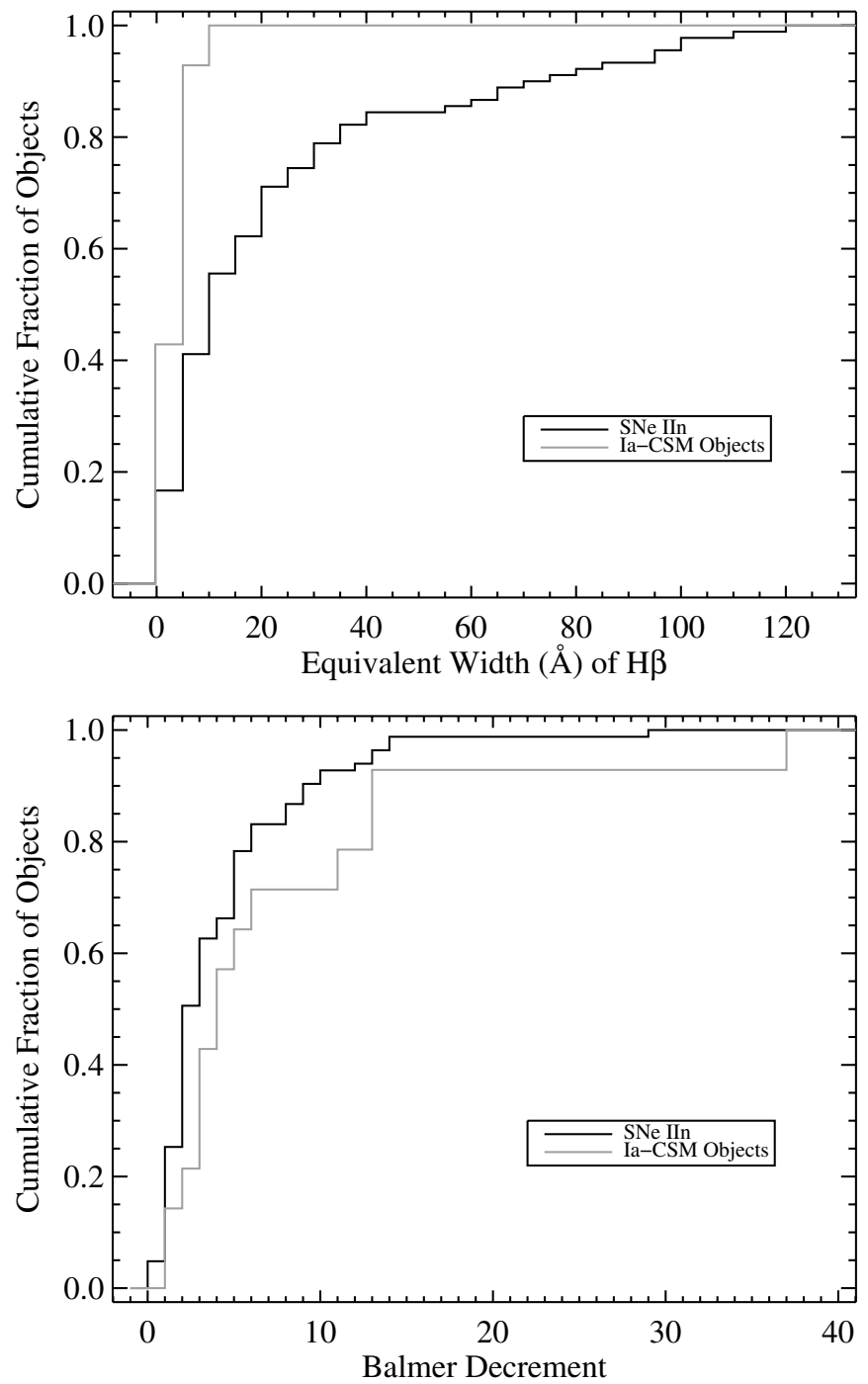

Figure 17. Cumulative fraction of $\mathrm{H} \beta$ EW (top) and $\mathrm{H} \alpha / \mathrm{H} \beta$ intensity ratio (Balmer decrement; bottom) for all SNe IIn from the Berkeley SN Group's database and PTF (black), and for SNe Ia-CSM with spectra presented herein (gray). The median values were used when there were multiple measurements for a given object.

\subsection{Host Galaxies of SNe Ia-CSM}

Prieto et al. (2007) found that the host galaxies of the first four discovered SNe Ia-CSM (SNe 1997cy, 1999E, 2002ic, and 2005gj) are all late-type galaxies (dwarf irregulars and late-type spirals) with star formation likely occurring within the last few hundred Myr. They also show that, with the exception of the host of SN 1999E, the hosts have low luminosities $(-19.1$ mag $<$ $M_{r}<-17.6 \mathrm{mag}$, similar to the Magellanic Clouds), which implies subsolar metallicities. The host of SN 1999E, however, is bright in the IR, shows a nuclear starburst, but is consistent with solar metallicity and the Milky Way (MW) luminosity (Allen et al. 1991; Prieto et al. 2007).

Using the NASA/IPAC Extragalactic Database (NED) and the SDSS Data Release 8 (DR8; Aihara et al. 2011), we find that the hosts of the four other non-PTF SNe Ia-CSM discussed in this work are also late-type galaxies. Three of the four have relatively low luminosities $\left(-19.3 \mathrm{mag}<M_{r}<-18.1 \mathrm{mag}\right)$, while the host of SN 2008J appears to be consistent with MW luminosity (much like the host of SN 1999E).

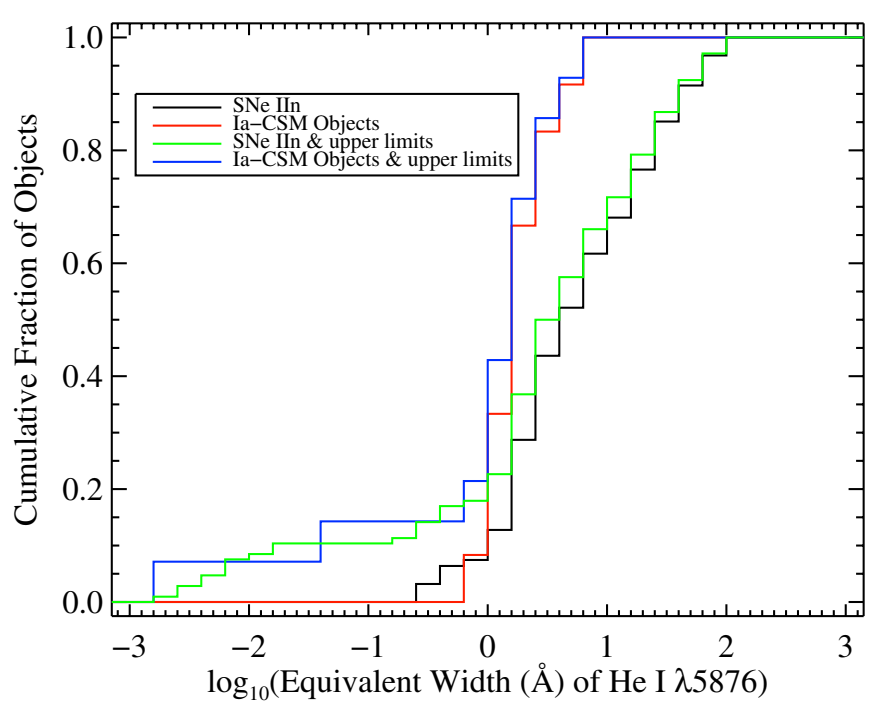

Figure 18. Cumulative fraction of $\mathrm{He}_{\mathrm{I}} \lambda 5876 \mathrm{EW}$ for all $\mathrm{SNe}$ IIn from the Berkeley SN Group's database and PTF (black), and for SNe Ia-CSM with spectra presented herein (red). Also shown are the He I $\lambda 5876 \mathrm{EWs}$ for SNe IaCSM including upper limits (blue) and for SNe IIn as well (green). The median EW value is used when there are multiple measurements for a given object.

Turning now to the PTF SNe Ia-CSM, PTF11kx has a latetype spiral host with luminosity comparable to that of the MW and slightly higher-than-solar metallicity (Dilday et al. 2012; Tremonti et al. 2004). Four of the seven newly discovered SNe Ia-CSM in PTF (PTF10htz, PTF10iuf, PTF11dsb, and PTF12hnr) are also found in what are likely late-type spiral hosts, all of which have luminosities similar to that of the MW (-20.6 mag $<M_{r}<-19.2 \mathrm{mag}$ ). The remaining three PTF SNe Ia-CSM (PTF10yni, PTF11hzx, and PTF12efc) do not have detectable hosts in SDSS DR8 (Aihara et al. 2011) or in our deep stacks of PTF search images. This implies that they are low-luminosity galaxies with $M_{r} \gtrsim-18 \mathrm{mag}$.

Thus, of the 16 SNe Ia-CSM discussed herein, all appear to have exploded in late-type galaxies. Nine of them are found in low-luminosity (and presumably, low-metallicity) hosts, including three hosts that are not detected by SDSS DR8 or PTF, while seven of them (including PTF11kx) are found in hosts roughly similar to the MW. Interestingly, when the hosts of PTF SNe Ia-CSM are compared to the hosts of SNe IIn from PTF, no significant differences are found. It has been shown in earlier work that $\mathrm{SNe}$ II in general have statistically the same hosts as SNe IIn (Kelly \& Kirshner 2012), and that SNe IIn and SNe IIP both trace recent (but not ongoing) star formation (Anderson et al. 2012). Furthermore, contrary to some expectations, Anderson et al. (2012) find that SNe IIn do not come from the youngest (and thus most massive) stars; instead, their progenitors are slightly older than those of SNe IIP. They also find that the association with host-galaxy $\mathrm{H} \alpha$ emission for $\mathrm{SNe}$ IIn is between that of other $\mathrm{SNe}$ II and $\mathrm{SNe}$ Ia, perhaps implying that some of their SNe IIn are actually SNe Ia-CSM which have lower-mass progenitors than any CCSN subtype. ${ }^{24}$

\section{CONCLUSIONS}

Running SNID on the Berkeley SN Group's database of SN IIn spectra, we have reidentified four SNe Ia-CSM that were previously known (SNe 2008J, 2008cg, 2011jb, and

\footnotetext{
${ }^{24}$ Note that none of the SNe Ia-CSM discussed herein were part of the
} Anderson et al. (2012) sample. 
CSS120327:110520-015205) but poorly studied. These are in addition to the four well-studied Ia-CSM objects (SNe 1997cy, 1999E, 2002ic, and 2005gj). Furthermore, SNID was run on all 63 SNe IIn discovered by PTF through 2012 August, and 7 new SNe Ia-CSM were uncovered. Armed with a sample of $15 \mathrm{SNe}$ Ia-CSM, in addition to PTF11kx (Dilday et al. 2012; Silverman et al. 2013), we investigate the unifying characteristics of this class of SN. Observable signatures of $\mathrm{SNe}$ Ia-CSM are as follows.

1. Peak absolute magnitudes of $-21.3 \mathrm{mag} \leqslant M_{R} \leqslant$ -19 mag are observed (somewhat more luminous than normal SNe Ia and the bulk of the SNe IIn population, but less luminous than superluminous $\mathrm{SNe}$ ) and relatively long rise times of $\sim 20-40$ days (as opposed to $\sim 18$ days for more normal SNe Ia).

2. SNID cross-correlations of optical spectra show that SNe Ia-CSM are spectroscopically homogeneous. The spectra consist of a diluted SN Ia spectrum, along with a relatively blue "quasi-continuum" from many blended lines of IGEs, strong and broad $\left(\sim 10,000 \mathrm{~km} \mathrm{~s}^{-1}\right)$ emission from the Ca II near-IR triplet, and are dominated by $\mathrm{H} \alpha$ emission with widths of $\sim 2000 \mathrm{~km} \mathrm{~s}^{-1}$.

3. The $\mathrm{H} \alpha$ profile shows strong fluctuations until $\sim 100-150$ days past maximum brightness, at which time the strength tends to increase with time. There are also extremely narrow (50-100 $\mathrm{km} \mathrm{s}^{-1}$ ) P-Cygni profiles present in $\mathrm{H} \alpha$, and after $\sim 75-100$ days past maximum a decrease in flux in the red wing is seen (often attributed to newly formed dust).

4. Weak He I and $\mathrm{H} \beta$ emission are seen, as compared to typical SNe IIn, and the $\mathrm{H} \alpha / \mathrm{H} \beta$ intensity ratio is large. This is likely caused by collisional excitation of hydrogen when the SN Ia-CSM ejecta overtake slower-moving, thin, moderately dense CSM shells.

5. Within the first few months after explosion, ultraviolet emission is seen, but no radio or X-ray emission is detected (although the range of upper limits derived is consistent with both upper limits and actual detections of SNe IIn at radio and X-ray wavelengths). Mid-IR emission is visible at $\sim 0.5-2$ yr past maximum brightness, and it is stronger than in typical SNe IIn or SNe Ia (which is further evidence of newly formed dust).

6. Using both rise times and X-ray upper limits, SNe Ia-CSM seem to have mass-loss rates of a few times $10^{-1} M_{\odot} \mathrm{yr}^{-1}$, though we caution that there are many assumptions that go into these calculations.

7. The hosts of SNe Ia-CSM all appear to be late-type spirals with either MW-like luminosities (and solar metallicities) or dwarf irregulars having luminosities similar to those of the Magellanic Clouds (and thus presumably subsolar metallicities).

While the above list describes attributes of the Ia-CSM objects, several of the items can also describe more typical SNe IIn. This brings up the possibility, noted previously (e.g., Anderson et al. 2012), that some subset of the SN IIn population is contaminated by SNe Ia-CSM. Perhaps there are simply some bona fide SNe IIn that share with SNe Ia-CSM many, but not all, of the observational characteristics listed above. It is also plausible that some SNe IIn that can be described by many of the items listed above are, in fact, SNe Ia-CSM where the CSM interaction is too strong or begins too early for any hint of an underlying SN Ia to be identified. On the other hand, at least some $\mathrm{SNe}$ IIn are actual core-collapse events since a massive progenitor star is detected (e.g., SN 2005gl; Gal-Yam \& Leonard 2009).

The existence of the SN Ia-CSM class of objects seems to argue that at least some SNe Ia arise from the SD channel, since a H-rich CSM is likely a result of that progenitor scenario (but see Shen et al. 2013). Detailed modeling of both the core collapse of massive stars and the thermonuclear explosion of WDs (with an assortment of binary companions) within various arrangements of CSM is beyond the scope of this paper, but we hope that future theoretical work on this subject will be informed by the observational results presented herein.

We thank K. Alatalo, T. Barlow, E. Bellm, B. Cobb, A. Cucchiara, M. Ganeshalingam, Y. Green, M. Hidas, L. Kewley, N. Konidaris, S. Lazarevic, N. Lee, D. Levitan, M. McCourt, K. Mooley, R. Mostardi, D. Perley, A. G. Riess, B. Sesar, R. Street, T. Treu, V. Viscomi, and X. Wang for their assistance with some of the observations and data reduction; B. Dilday, O. Fox, and L. Wang for helpful discussions; and D. Balam, M. Stritzinger, J. Vinko, and J. C. Wheeler for providing unpublished spectra of possible SNe Ia-CSM. We are grateful to the staffs at the Isaac Newton Group of Telescopes and the Lick, Keck, Palomar, and Kitt Peak National Observatories for their support. Some of the data presented herein were obtained at the W. M. Keck Observatory, which is operated as a scientific partnership among the California Institute of Technology, the University of California, and the National Aeronautics and Space Administration (NASA); the observatory was made possible by the generous financial support of the W. M. Keck Foundation. The authors recognize and acknowledge the very significant cultural role and reverence that the summit of Mauna Kea has always had within the indigenous Hawaiian community; we are most fortunate to have the opportunity to conduct observations from this mountain. This research has made use of the NASA/IPAC Extragalactic Database (NED) which is operated by the Jet Propulsion Laboratory, California Institute of Technology, under contract with NASA. Funding for SDSSIII has been provided by the Alfred P. Sloan Foundation, the Participating Institutions, the National Science Foundation, and the U.S. Department of Energy Office of Science. The SDSSIII Web site is http://www.sdss3.org/. Supernova research by A.V.F.'s group at UC Berkeley is supported by Gary and Cynthia Bengier, the Richard and Rhoda Goldman Fund, the Christopher R. Redlich Fund, the TABASGO Foundation, and NSF grants AST-0908886 and AST-1211916. Work by A.G.-Y. and his group is supported by grants from the ISF, BSF, GIF, Minerva, an FP7/ERC grant, and the Helen and Martin Kimmel Award for Innovative Investigation. M.M.K. acknowledges generous support from a Hubble Fellowship and a Carnegie-Princeton Fellowship.

\section{REFERENCES}

Aihara, H., Allende Prieto, C., An, D., et al. 2011, ApJS, 195, 26

Aldering, G., Antilogus, P., Bailey, S., et al. 2006, ApJ, 650, 510

Allen, D. A., Norris, R. P., Meadows, V. S., \& Roche, P. F. 1991, MNRAS, 248,528

Anderson, J. P., Habergham, S. M., James, P. A., \& Hamuy, M. 2012, MNRAS, 424, 1372

Arcavi, I., Sternberg, A., Yaron, O., et al. 2010a, ATel, 2685, 1

Arcavi, I., Xu, D., Matheson, T., et al. 2010b, ATel, 3027, 1

Benetti, S., Cappellaro, E., Turatto, M., et al. 2006, ApJL, 653, L129

Blondin, S., \& Calkins, M. 2008, CBET, 1368, 1

Blondin, S., Prieto, J. L., Patat, F., et al. 2009, ApJ, 693, 207 
Blondin, S., \& Tonry, J. L. 2007, ApJ, 666, 1024

Bloom, J. S., Kasen, D., Shen, K. J., et al. 2012, ApJL, 744, L17

Brown, P. J., Dawson, K. S., de Pasquale, M., et al. 2012, ApJ, 753, 22

Chandra, P., \& Soderberg, A. 2008, ATel, 1594, 1

Chevalier, R. A., \& Fransson, C. 1994, ApJ, 420, 268

Chugai, N. N., Chevalier, R. A., \& Lundqvist, P. 2004, MNRAS, 355, 627

Chugai, N. N., \& Yungelson, L. R. 2004, AstL, 30, 65

Deng, J., Kawabata, K. S., Ohyama, Y., et al. 2004, ApJL, 605, L37

Dilday, B., Howell, D. A., Cenko, S. B., et al. 2012, Sci, 337, 942

Drake, A. J., Djorgovski, S. G., Mahabal, A. A., et al. 2010, ATel, 2978, 1

Drake, A. J., Garcia-Alvarez, D., Djorgovski, S. G., et al. 2012, ATel, 4081, 1

Drake, S. A., \& Ulrich, R. K. 1980, BAAS, 12, 798

Filippenko, A. V. 1997, ARA\&A, 35, 309

Filippenko, A. V. 2000, in AIP Conf. Proc. 522, Cosmic Explosions: Tenth Astrophysics Conference, ed. S. S. Holt \& W. W. Zhang (Melville, NY: AIP), 123

Filippenko, A. V., Silverman, J. M., Mostardi, R., \& Griffith, C. V. 2008, CBET, 1420,1

Foley, R. J., Challis, P. J., Filippenko, A. V., et al. 2012a, ApJ, 744, 38

Foley, R. J., Simon, J. D., Burns, C. R., et al. 2012b, ApJ, 752, 101

Fox, O. D., Chevalier, R. A., Skrutskie, M. F., et al. 2011, ApJ, 741, 7

Gal-Yam, A. 2012, Sci, 337, 927

Gal-Yam, A., \& Leonard, D. C. 2009, Natur, 458, 865

Gal-Yam, A., Nugent, P., Quimby, R., et al. 2011, ATel, 3403, 1

Ganeshalingam, M., Li, W., \& Filippenko, A. V. 2011, MNRAS, 416, 2607

Ganeshalingam, M., Li, W., Filippenko, A. V., et al. 2010, ApJS, 190, 418

Garavini, G., Folatelli, G., Goobar, A., et al. 2004, AJ, 128, 387

Germany, L. M., Reiss, D. J., Sadler, E. M., Schmidt, B. P., \& Stubbs, C. W. 2000, ApJ, 533, 320

Hamuy, M., Phillips, M. M., Suntzeff, N. B., et al. 2003, Natur, 424, 651

Howell, D. A. 2011, NatCo, 2, 350

Howell, D. A., Sullivan, M., Nugent, P. E., et al. 2006, Natur, 443, 308

Iben, I., Jr., \& Tutukov, A. V. 1984, ApJS, 54, 335

Immler, S., Brown, P. J., Filippenko, A. V., \& Pooley, D. 2007, ATel, 1290, 1

Immler, S., Filippenko, A. V., \& Pooley, D. 2008, ATel, 1598, 1

Immler, S., Petre, R., \& Brown, P. 2005, IAUC, 8633, 2

Kankare, E., Mattila, S., \& Pastorello, A. 2011, CBET, 2947, 2

Kelly, P. L., \& Kirshner, R. P. 2012, ApJ, 759, 107

Kiewe, M., Gal-Yam, A., Arcavi, I., et al. 2012, ApJ, 744, 10

Kotak, R., Meikle, W. P. S., Adamson, A., \& Leggett, S. K. 2004, MNRAS, 354, L13

Law, N. M., Kulkarni, S. R., Dekany, R. G., et al. 2009, PASP, 121, 1395

Li, W., Filippenko, A. V., Treffers, R. R., et al. 2001, ApJ, 546, 734

Li, W., Leaman, J., Chornock, R., et al. 2011, MNRAS, 412, 1441

Moore, K., \& Bildsten, L. 2012, ApJ, 761, 182
Nugent, P. E., Sullivan, M., Cenko, S. B., et al. 2011, Natur, 480, 344

Ofek, E. O., Laher, R., Law, N., et al. 2012a, PASP, 124, 62

Ofek, E. O., Laher, R., Surace, J., et al. 2012b, PASP, 124, 854

Ofek, E. O., Sullivan, M., Cenko, S. B., et al. 2013, Natur, 494, 65

Patat, F., Chandra, P., Chevalier, R., et al. 2007, Sci, 317, 924

Peek, J. E. G., \& Graves, G. J. 2010, ApJ, 719, 415

Phillips, M. M. 1993, ApJL, 413, L105

Pooley, D., Lewin, W. H. G., Fox, D. W., et al. 2002, ApJ, 572, 932

Prieto, J. L., Garnavich, P. M., Phillips, M. M., et al. 2007, AJ, submitted (arXiv:0706.4088)

Quimby, R. M., Yuan, F., Akerlof, C., \& Wheeler, J. C. 2013, MNRAS, 431,912

Rau, A., Kulkarni, S. R., Law, N. M., et al. 2009, PASP, 121, 1334

Rigon, L., Turatto, M., Benetti, S., et al. 2003, MNRAS, 340, 191

Scalzo, R. A., Aldering, G., Antilogus, P., et al. 2010, ApJ, 713, 1073

Schlegel, D. J., Finkbeiner, D. P., \& Davis, M. 1998, ApJ, 500, 525

Shen, K. J., Guillochon, J., \& Foley, R. J. 2013, ApJL, in press (arXiv:1302.2916)

Silverman, J. M., Foley, R. J., Filippenko, A. V., et al. 2012a, MNRAS, 425,1789

Silverman, J. M., Ganeshalingam, M., Cenko, S. B., et al. 2012b, ApJL, $756, \mathrm{~L} 7$

Silverman, J. M., Ganeshalingam, M., Li, W., et al. 2011, MNRAS, 410, 585

Silverman, J. M., Nugent, P. E., Gal-Yam, A., et al. 2013, ApJ, in press (arXiv:1303.7234)

Simon, J. D., Gal-Yam, A., Gnat, O., et al. 2009, ApJ, 702, 1157

Smith, N., Li, W., Miller, A. A., et al. 2011a, ApJ, 732, 63

Smith, N., Li, W., Silverman, J. M., Ganeshalingam, M., \& Filippenko, A. V. 2011b, MNRAS, 415, 773

Smith, N., Silverman, J. M., Filippenko, A. V., et al. 2012, AJ, 143, 17

Soderberg, A. M., \& Frail, D. A. 2005, ATel, 663, 1

Sternberg, A., Gal-Yam, A., Simon, J. D., et al. 2011, Sci, 333, 856

Stritzinger, M., Folatelli, G., \& Morrell, N. 2008, CBET, 1218, 1

Strolger, L., Smith, R. C., Suntzeff, N. B., et al. 2002, AJ, 124, 2905

Taddia, F., Stritzinger, M. D., Phillips, M. M., et al. 2012, A\&A, 545, L7

Taubenberger, S., Benetti, S., Childress, M., et al. 2011, MNRAS, 412, 2735

Tremonti, C. A., Heckman, T. M., Kauffmann, G., et al. 2004, ApJ, 613, 898

Trundle, C., Kotak, R., Vink, J. S., \& Meikle, W. P. S. 2008, A\&A, 483, L47

Turatto, M., Suzuki, T., Mazzali, P. A., et al. 2000, ApJL, 534, L57

Wang, L., Baade, D., Höflich, P., et al. 2004, ApJL, 604, L53

Webbink, R. F. 1984, ApJ, 277, 355

Whelan, J., \& Iben, I., Jr. 1973, ApJ, 186, 1007

Wood-Vasey, W. M., Wang, L., \& Aldering, G. 2004, ApJ, 616, 339

Xu, Y., McCray, R., Oliva, E., \& Randich, S. 1992, ApJ, 386, 181

Yamanaka, M., Kawabata, K. S., Kinugasa, K., et al. 2009, ApJL, 707, L118

Yaron, O., \& Gal-Yam, A. 2012, PASP, 124, 668

Zampieri, L., Mucciarelli, P., Pastorello, A., et al. 2005, MNRAS, 364, 1419 